\title{
A GENERALIZATION OF GELFAND-NAIMARK-STONE DUALITY TO COMPLETELY REGULAR SPACES
}

\author{
G. BEZHANISHVILI, P. J. MORANDI, AND B. OLBERDING
}

\begin{abstract}
Gelfand-Naimark-Stone duality establishes a dual equivalence between the cat-

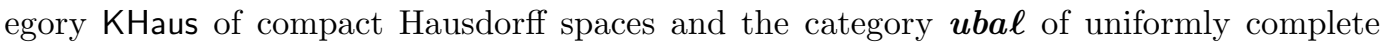
bounded archimedean $\ell$-algebras. We extend this duality to the category CReg of completely regular spaces. This we do by first introducing basic extensions of bounded archimedean $\ell$-algebras and generalizing Gelfand-Naimark-Stone duality to a dual equivalence between the category ubasic of uniformly complete basic extensions and the category Comp of compactifications of completely regular spaces. We then introduce maximal basic extensions and prove that the subcategory mbasic of ubasic consisting of maximal basic extensions is dually equivalent to the subcategory SComp of Comp consisting of Stone-Čech compactifications. This yields the desired dual equivalence for completely regular spaces since CReg is equivalent to SComp.
\end{abstract}

\section{INTRODUCTION}

Let CReg be the category of completely regular spaces and continuous maps, and let KHaus be its full subcategory consisting of compact Hausdorff spaces. Let also $\boldsymbol{b} \boldsymbol{a} \boldsymbol{\ell}$ be the category of bounded archimedean $\boldsymbol{\ell}$-algebras and unital $\ell$-algebra homomorphisms, and let $\boldsymbol{u} \boldsymbol{b a} \boldsymbol{\ell}$ be its full subcategory consisting of uniformly complete objects in $\boldsymbol{b a} \boldsymbol{\ell}$ (see Section 2 for definitions). There is a contravariant functor $C^{*}: \mathrm{CReg} \rightarrow \boldsymbol{b} \boldsymbol{a} \boldsymbol{\ell}$ sending a completely regular space $X$ to the $\ell$-ring $C^{*}(X)$ of bounded continuous real-valued functions, and a contravariant functor $Y: \boldsymbol{b} \boldsymbol{a} \boldsymbol{\ell} \rightarrow$ CReg sending $A \in \boldsymbol{b} \boldsymbol{a} \boldsymbol{\ell}$ to the space of maximal $\ell$-ideals. The functors $C^{*}$ and $Y$ define a contravariant adjunction between CReg and $\boldsymbol{b a} \boldsymbol{\ell}$ such that $C^{*}(X) \in \boldsymbol{u b a} \boldsymbol{\ell}$ for each $X \in \mathrm{KH}$ aus and $Y(A) \in \mathrm{KH}$ aus for each $A \in \boldsymbol{b} \boldsymbol{a} \boldsymbol{\ell}$. Thus, the contravariant adjunction between CReg and $\boldsymbol{b} \boldsymbol{a} \boldsymbol{\ell}$ restricts to a dual equivalence between KHaus and $\boldsymbol{u} \boldsymbol{b} \boldsymbol{a} \boldsymbol{\ell}$. This dual equivalence is known as Gelfand-Naimark-Stone duality (see [11, 18, 12, 14, 2]). We note that if $X \in$ KHaus, then every continuous real-valued function on $X$ is bounded. Therefore, $C^{*}(X)$ is equal to the $\ell$-ring $C(X)$ of all continuous real-valued functions. Thus, the functor $C^{*}:$ CReg $\rightarrow \boldsymbol{b} \boldsymbol{a} \boldsymbol{\ell}$ restricts to the functor $C:$ KHaus $\rightarrow \boldsymbol{u} \boldsymbol{b} \boldsymbol{a} \boldsymbol{\ell}$, and we arrive at the following commutative diagram.

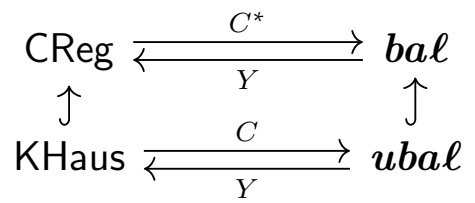

2010 Mathematics Subject Classification. 54D15; 54D35; 54C30; 06F25; 13J25.

Key words and phrases. Completely regular space; compactification, continuous real-valued function; $\ell$ algebra. 
The purpose of this article is to extend Gelfand-Naimark-Stone duality to completely regular spaces. For this it is not sufficient to only work with the $\ell$-ring $C^{*}(X)$. The space of maximal $\ell$-ideals of $C^{*}(X)$ is the Stone-Čech compactification $\beta X$, and hence $X$ is not recoverable as the space of maximal $\ell$-ideals of $C^{*}(X)$. To recover $X$ additional data is required, which we show can be provided by also working with the $\ell$-ring $B(X)$ of bounded functions on $X$. The idempotents of $B(X)$ are exactly the characteristic functions of subsets of $X$, so the boolean algebra $\operatorname{Id}(B(X))$ of idempotents of $B(X)$ is isomorphic to the powerset $\wp(X)$. The singletons $\{x\}$ are the atoms of $\wp(X)$, which correspond to the primitive idempotents of $B(X)$. Therefore, $X$ is in bijective correspondence with the primitive idempotents of $B(X)$. Thus, to recover the topology on $X$ it is sufficient to give an algebraic description of the embedding $C^{*}(X) \rightarrow B(X)$. Since $C^{*}(X)$ is isomorphic to $C(\beta X)$, it suffices to give an algebraic description of the monomorphism $C(\beta X) \rightarrow B(X)$ arising from the embedding $X \rightarrow \beta X$. More generally, given a compactification $e: X \rightarrow Y$, we will give an algebraic description of the monomorphism $C(Y) \rightarrow B(X)$ arising from $e$ (it is a monomorphism since $e[X]$ is dense in $Y)$.

For this we will first characterize the algebras $B(X)$ as Dedekind complete (bounded) archimedean $\ell$-algebras in which the boolean algebra of idempotents is atomic. We term such algebras basic algebras, and prove that the category balg of basic algebras and the unital $\ell$-algebra homomorphisms between them that are normal (meaning that they preserve all existing joins, and hence meets) is dually equivalent to the category Set of sets and functions. This provides a ring-theoretic version of Tarski duality between Set and the category CABA of complete and atomic boolean algebras and complete boolean homomorphisms.

We next extend the focus from algebras in $\boldsymbol{b a} \boldsymbol{\ell}$ to what we call basic extensions. These are extensions $\alpha: A \rightarrow B$ such that $A \in \boldsymbol{b a} \boldsymbol{\ell}, B \in \boldsymbol{b a l g}$, and $\alpha[A]$ is join-meet dense in $B$. Each compactification $e: X \rightarrow Y$ gives rise to the basic extension $e^{b}: C(Y) \rightarrow B(X)$. In Theorem 6.3 we prove that this correspondence extends to a dual adjunction between the category Comp of compactifications and the category basic of basic extensions, which restricts to a dual equivalence between Comp and the full subcategory ubasic of basic consisting of uniformly complete basic extensions.

We further consider the full subcategory SComp of Comp consisting of Stone-Čech compactifications, and prove that the dual equivalence between Comp and ubasic restricts to a dual equivalence between SComp and the full subcategory mbasic of ubasic consisting of maximal basic extensions, which can be characterized as those uniformly complete basic extensions $\alpha: A \rightarrow B$ for which the only elements of $B$ that are both a join and meet of elements from $\alpha[A]$ are the elements of $\alpha[A]$ itself. Since the category CReg of completely regular spaces is equivalent to SComp, we conclude that the maximal basic extensions provide an algebraic counterpart of the completely regular spaces.

This article can be viewed as a ring-theoretic companion to our article [6], in which we show that the category of completely regular spaces is dually equivalent to the category of what we call maximal de Vries extensions in [6], a certain class of extensions of complete Boolean algebras equipped with a proximity relation. In so doing we extend de Vries duality to completely regular spaces in direct analogy with how we extend Gelfand-Naimark-Stone 
duality to completely regular spaces in the present paper. In a future paper, we will make the analogy between these two settings more precise. For the present, however, these two dualities for completely regular spaces remain independent of each other in our approaches.

The article is organized as follows. In Section 2 we recall Gelfand-Naimark-Stone duality and describe its restriction to the full subcategories of KHaus consisting of Stone spaces and extremally disconnected spaces. Section 3 introduces basic algebras and their fundamental properties. We prove that balg is dually equivalent to Set, which is a ring-theoretic version of Tarski duality between CABA and Set. In Section 4 we define basic extensions and uniformly complete basic extensions, and show that the category ubasic of uniformly complete basic extensions is a reflective subcategory of the category basic of basic extensions. We also define a functor from Comp to basic, and show that it lands in ubasic. In Section 5 we produce a functor going the other way, from basic to Comp. With these functors in place, we show in Section 6 that there is a dual adjunction between basic and Comp, which restricts to a dual equivalence between ubasic and Comp. Finally, building on the previous sections, we obtain in Section 7 our generalization of Gelfand-Naimark-Stone duality between the category CReg of completely regular spaces and the category mbasic of maximal basic extensions, a special class of basic extensions that we describe in detail in Section 7.

\section{Gelfand-Naimark-Stone duality}

In this section we recall Gelfand-Naimark-Stone duality. This requires recalling a number of basic facts about ordered rings and algebras. For general references we use [7, 12, 14, 15]. For a detailed study of the category $\boldsymbol{b a} \boldsymbol{\ell}$, which plays a central role for our purposes, we refer to [2].

For a completely regular space $X$, let $C(X)$ be the ring of continuous real-valued functions, and let $C^{*}(X)$ be the subring of $C(X)$ consisting of bounded functions. We note that if $X$ is compact, then $C^{*}(X)=C(X)$. There is a natural partial order $\leq$ on $C(X)$ lifted from $\mathbb{R}$. Then $C^{*}(X)$ with the restriction of $\leq$ is a bounded archimedean $\ell$-algebra, where we recall that

- A ring $A$ with a partial order $\leq$ is an $\ell$-ring (lattice-ordered ring) if $(A, \leq)$ is a lattice, $a \leq b$ implies $a+c \leq b+c$ for each $c$, and $0 \leq a, b$ implies $0 \leq a b$.

- An $\ell$-ring $A$ is bounded if for each $a \in A$ there is $n \in \mathbb{N}$ such that $a \leq n \cdot 1$ (that is, 1 is a strong order unit).

- An $\ell$-ring $A$ is archimedean if for each $a, b \in A$, whenever $n a \leq b$ for each $n \in \mathbb{N}$, then $a \leq 0$.

- An $\ell$-ring $A$ is an $\ell$-algebra if it is an $\mathbb{R}$-algebra and for each $0 \leq a \in A$ and $0 \leq r \in \mathbb{R}$ we have $r a \geq 0$.

- Let $\boldsymbol{b} \boldsymbol{a} \boldsymbol{\ell}$ be the category of bounded archimedean $\ell$-algebras and unital $\ell$-algebra homomorphisms.

Convention 2.1. For a continuous map $\varphi: X \rightarrow Y$ between completely regular spaces let $\varphi^{*}: C^{*}(Y) \rightarrow C^{*}(X)$ be given by $\varphi^{*}(f)=f \circ \varphi$. 
Then $\varphi^{*}$ is a unital $\ell$-algebra homomorphism, and we have a contravariant functor $C^{*}$ : CReg $\rightarrow \boldsymbol{b a} \boldsymbol{\ell}$ which sends each $X \in \mathrm{CReg}$ to the $\ell$-algebra $C^{*}(X)$, and each continuous map $\varphi: X \rightarrow Y$ to the unital $\ell$-algebra homomorphism $\varphi^{*}: C^{*}(Y) \rightarrow C^{*}(X)$. We denote the restriction of $C^{*}$ to KHaus by $C$ since for $X \in$ KHaus we have $C^{*}(X)=C(X)$.

The functor $C^{*}$ has a contravariant adjoint which is defined as follows. For $A \in \boldsymbol{b} \boldsymbol{a} \boldsymbol{\ell}$ and $a \in A$, we recall that the absolute value of $a$ is defined as $|a|=a \vee(-a)$, that an ideal $I$ of $A$ is an $\ell$-ideal if $|a| \leq|b|$ and $b \in I$ imply $a \in I$, and that $\ell$-ideals are exactly the kernels of $\ell$-algebra homomorphisms. Let $Y_{A}$ be the space of maximal $\ell$-ideals of $A$, whose closed sets are exactly sets of the form

$$
Z_{\ell}(I)=\left\{M \in Y_{A} \mid I \subseteq M\right\}
$$

where $I$ is an $\ell$-ideal of $A$. The space $Y_{A}$ is often referred to as the Yosida space of $A$, and it is well known that $Y_{A} \in$ KHaus.

Convention 2.2. For a unital $\ell$-algebra homomorphism $\alpha: A \rightarrow B$ let $\alpha_{\star}: Y_{B} \rightarrow Y_{A}$ be given by $\alpha_{*}(M)=\alpha^{-1}(M)$.

Then $\alpha_{*}$ is continuous, and we have a contravariant functor $Y: \boldsymbol{b} \boldsymbol{a} \boldsymbol{\ell} \rightarrow$ CReg which sends each $A \in \boldsymbol{b} \boldsymbol{a} \boldsymbol{\ell}$ to the compact Hausdorff space $Y_{A}$, and each unital $\ell$-algebra homomorphism $\alpha: A \rightarrow B$ to the continuous map $\alpha_{\star}: Y_{B} \rightarrow Y_{A}$.

For $A \in \boldsymbol{b a} \boldsymbol{\ell}$ and $X \in \mathrm{CReg}$, we have $\operatorname{hom}_{\boldsymbol{b a} \boldsymbol{\ell}}\left(A, C^{*}(X)\right) \simeq \operatorname{hom}_{\mathrm{CReg}}\left(X, Y_{A}\right)$. Thus, $Y$ and $C^{*}$ define a contravariant adjunction between $\boldsymbol{b a} \boldsymbol{\ell}$ and CReg. As we already pointed out, $Y_{A} \in$ KHaus. In fact, each compact Hausdorff space is homeomorphic to $Y_{A}$ for some $A \in \boldsymbol{b a} \boldsymbol{\ell}$. To see this, for $X \in \mathrm{CReg}$, associate with each $x \in X$ the maximal $\ell$-ideal

$$
M_{x}:=\left\{f \in C^{*}(X) \mid f(x)=0\right\} .
$$

Then $\xi_{X}: X \rightarrow Y_{C^{*}(X)}$ given by $\xi_{X}(x)=M_{x}$ is an embedding, and it is a homeomorphism iff $X$ is compact.

To describe which objects of $\boldsymbol{b a} \boldsymbol{\ell}$ are isomorphic to $C^{*}(X)$ for some $X$, we observe that for a maximal $\ell$-ideal $M$ of $A \in \boldsymbol{b} \boldsymbol{a} \boldsymbol{\ell}$, we have $A / M \simeq \mathbb{R}$. Therefore, with each $a \in A$, we can associate $f_{a} \in C\left(Y_{A}\right)$ given by $f_{a}(M)=a+M$. Then $\zeta_{A}: A \rightarrow C\left(Y_{A}\right)$ given by $\zeta_{A}(a)=f_{a}$ is a unital $\ell$-algebra homomorphism, which is a monomorphism since the intersection of maximal $\ell$-ideals is 0 . It is an isomorphism iff the norm on $A$ defined by

$$
\|a\|=\inf \{r \in \mathbb{R}|| a \mid \leq r\}
$$

is complete. In such a case we call $A$ uniformly complete, and denote the full subcategory of $\boldsymbol{b a} \boldsymbol{\ell}$ consisting of uniformly complete $\ell$-algebras by $\boldsymbol{u} \boldsymbol{b a} \boldsymbol{\ell}$. Thus, $A \in \boldsymbol{u} \boldsymbol{b a} \boldsymbol{\ell}$ iff $A$ is isomorphic to $C^{*}(X)$ for some $X \in$ KHaus. Consequently, the contravariant adjunction $\left(C^{*}, Y\right)$ between CReg and $\boldsymbol{b} \boldsymbol{a} \boldsymbol{\ell}$ restricts to a dual equivalence $(C, Y)$ between KHaus and $\boldsymbol{u} \boldsymbol{b} \boldsymbol{a} \boldsymbol{\ell}$, and we arrive at the following celebrated result:

Theorem 2.3 (Gelfand-Naimark-Stone duality). The categories KHaus and uba $\boldsymbol{\ell}$ are dually equivalent, and the dual equivalence is established by the functors $C$ and $Y$.

Remark 2.4. While Stone worked with real-valued functions, Gelfand and Naimark worked with complex-valued functions. As a result, Gelfand-Naimark duality is between KHaus and 
the category $\mathrm{CC}^{*} \mathrm{Alg}$ of commutative $C^{*}$-algebras. However, $\boldsymbol{u} \boldsymbol{b a} \boldsymbol{\ell}$ is equivalent to $\mathrm{CC}^{*} \mathrm{Alg}$, and the equivalence is established by the following functors. The functor $\boldsymbol{u b a} \boldsymbol{\ell} \rightarrow \mathrm{CC}^{*} \mathrm{Alg}$ associates to each $A \in \boldsymbol{u} \boldsymbol{b a} \boldsymbol{\ell}$ its complexification $A \otimes_{\mathbb{R}} \mathbb{C}$; and the functor $\mathrm{CC}^{*} \mathrm{Alg} \rightarrow \boldsymbol{u b a} \boldsymbol{\ell}$ associates to each $A \in \mathrm{CC}^{*} \mathrm{Alg}$ its subalgebra of self-adjoint elements; for further details see, e.g., [2, Sec. 7].

We recall that a subset of a topological space $X$ is clopen if it is closed and open, that $X$ is zero-dimensional if it has a basis of clopens, and that $X$ is extremally disconnected if the closure of each open is clopen. Zero-dimensional compact Hausdorff spaces are usually referred to as Stone spaces because, by the celebrated Stone duality, they provide the dual counterpart of boolean algebras.

Let Stone be the full subcategory of KHaus consisting of Stone spaces, and let ED be the full subcategory of Stone consisting of extremally disconnected objects of KHaus. GelfandNaimark-Stone duality yields interesting restrictions to Stone and ED.

We recall that a commutative ring $A$ is a clean ring provided each element of $A$ is the

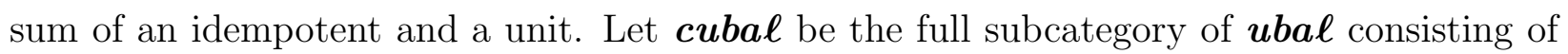
clean rings. By [1, Thm. 2.5], a compact Hausdorff space $X$ is a Stone space iff $C(X)$ is a clean ring. This together with Gelfand-Naimark-Stone duality yields:

Corollary 2.5. The categories Stone and cubal are dually equivalent, and the dual equivalence is established by restricting the functors $C$ and $Y$.

We recall that $A \in \boldsymbol{b} \boldsymbol{a} \boldsymbol{\ell}$ is Dedekind complete if each subset of $A$ bounded above has a least upper bound, and hence each subset bounded below has a greatest lower bound. Let $\boldsymbol{d} \boldsymbol{b} \boldsymbol{a} \boldsymbol{\ell}$ be the full subcategory of $\boldsymbol{b} \boldsymbol{a} \boldsymbol{\ell}$ consisting of Dedekind complete objects in $\boldsymbol{b} \boldsymbol{a} \boldsymbol{\ell}$. By [3. Thm. 3.3], $A \in \boldsymbol{b} \boldsymbol{a} \boldsymbol{\ell}$ is Dedekind complete iff $A \in \boldsymbol{u} \boldsymbol{b} \boldsymbol{a} \boldsymbol{\ell}$ and $A$ is a Baer ring, where we recall that a commutative ring is a Baer ring provided each annihilator ideal is generated by an idempotent. Consequently, $\boldsymbol{d} \boldsymbol{b a} \boldsymbol{\ell}$ is a full subcategory of $\boldsymbol{c u b a} \boldsymbol{\ell}$. By the Stone-Nakano theorem [18, 19, 16], for $X \in \mathrm{KHaus}$ we have $C(X)$ is Dedekind complete iff $X \in \mathrm{ED}$. This together with Gelfand-Naimark-Stone duality yields:

Corollary 2.6. The categories ED and dbal are dually equivalent, and the dual equivalence is established by restricting the functors $C$ and $Y$.

Let BA be the category of boolean algebras and boolean homomorphisms, and let CBA be the full subcategory of BA consisting of complete boolean algebras. By Stone duality for boolean algebras, BA is dually equivalent to Stone and CBA is dually equivalent to ED. Thus, we arrive at the following diagram: 


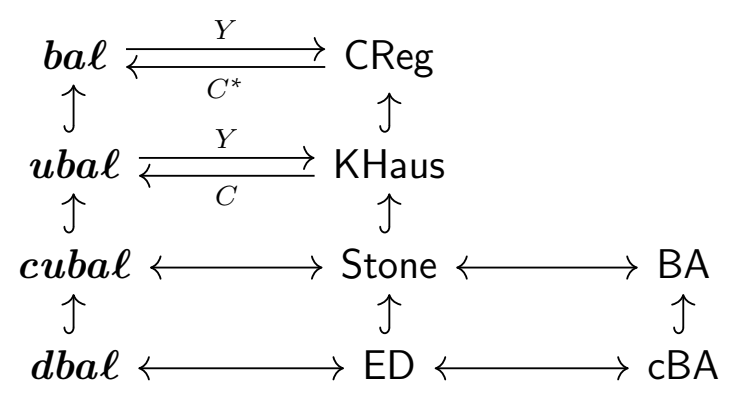

Our goal is to generalize Gelfand-Naimark-Stone duality to compactifications and completely regular spaces. To achieve this, we require new concepts of basic algebras and basic extensions.

\section{BASIC ALGEBRAS AND A RING-THEORETIC VERSION OF TARSKI DUALITY}

Tarski duality establishes a dual equivalence between the category Set of sets and functions and the category CABA of complete and atomic boolean algebras and complete boolean homomorphisms. The functor $\wp:$ Set $\rightarrow$ CABA sends a set $X$ to its powerset $\wp(X)$, and a function $\varphi: X \rightarrow Y$ to the boolean homomorphism $\varphi^{-1}: \wp(Y) \rightarrow \wp(X)$. The functor At $: \mathrm{CABA} \rightarrow$ Set sends $B \in \mathrm{CABA}$ to the set $\operatorname{At}(B)$ of atoms of $B$. If $\sigma: B \rightarrow C$ is a complete boolean homomorphism, then At sends $\sigma$ to $\sigma_{*}: \operatorname{At}(C) \rightarrow \operatorname{At}(B)$, defined by $\sigma_{*}(c)=\bigwedge\{b \in B \mid c \leq \sigma(b)\}$.

We next use Corollary 2.6 to give a ring-theoretic version of Tarski duality. For $A \in \boldsymbol{b a} \boldsymbol{\ell}$, let $\operatorname{Id}(A)$ be the boolean algebra of idempotents of $A$. We recall that an idempotent $e$ of $A$ is primitive if $e \neq 0$ and $0 \leq f \leq e$ for some $f \in \operatorname{Id}(A)$ implies $f=0$ or $f=e$. Thus, primitive idempotents are exactly the atoms of the boolean algebra $\operatorname{Id}(A)$. Let $\operatorname{Prim}(A)$ be the set of primitive idempotents of $A$.

Let $A \in \boldsymbol{d} \boldsymbol{b a} \boldsymbol{\ell}$. Then $\operatorname{Id}(A)$ is complete. By Corollary 2.6, $Y_{A} \in \mathrm{ED}$ and $\alpha: A \rightarrow C\left(Y_{A}\right)$ is an isomorphism. Moreover, $\operatorname{Id}(A)$ is isomorphic to the boolean algebra $\operatorname{Clop}\left(Y_{A}\right)$ of clopen subsets of $Y_{A}$. Primitive idempotents then correspond to isolated points of $Y_{A}$.

Convention 3.1. Let $X_{A}$ be the set of isolated points of $Y_{A}$.

As follows from the next lemma, the correspondence between primitive idempotents of $A$ and isolated points of $Y_{A}$ is obtained by associating with each primitive idempotent $e$ the maximal $\ell$-ideal $(1-e) A$.

Lemma 3.2. Let $A \in \boldsymbol{d b a} \ell$ and $0 \neq e \in \operatorname{Id}(A)$. The following are equivalent.

(1) $e$ is a primitive idempotent of $A$.

(2) $(1-e) A$ is a maximal $\ell$-ideal of $A$.

(3) For each $a \in A$, there is $r \in \mathbb{R}$ such that ae $=$ re.

Consequently, $X_{A}=\{(1-e) A \mid e \in \operatorname{Prim}(A)\}$.

Proof. $(1) \Rightarrow(2)$. This is proved in [5, Lem. 4.1]. 
A GENERALIZATION OF GELFAND-NAIMARK-STONE DUALITY

$(2) \Rightarrow(3)$. Since $(1-e) A$ is a maximal $\ell$-ideal, $A /(1-e) A \simeq \mathbb{R}$. Therefore, $a+(1-e) A=$ $r+(1-e) A$ for some $r \in \mathbb{R}$. So $a-r \in(1-e) A$. Since $1-e$ is an idempotent, this yields that $(a-r)(1-e)=(a-r)$. Thus, $(a-r) e=0$, and hence $a e=r e$.

$(3) \Rightarrow(1)$. Let $f \in \operatorname{Id}(A)$ and $f \leq e$. There is $r \in \mathbb{R}$ such that $f e=r e$. Since $f \leq e$ and $e, f \in \operatorname{Id}(A)$, we have $f=e f=r e$. Therefore, $r=0$ or $r=1$. Consequently, $f=0$ or $f=e$, proving that $e$ is primitive.

To prove the last statement of the lemma, since $A \in \boldsymbol{d} \boldsymbol{b a} \boldsymbol{\ell}$, we have $A \cong C\left(Y_{A}\right)$. Therefore, idempotents of $A$ correspond to characteristic functions of clopens of $Y_{A}$. So if $e \in \operatorname{Id}(A)$, then the sets $Z_{\ell}(e)$ and $Z_{\ell}(1-e)$ are complementary clopens. Now suppose $e$ is a primitive idempotent. By the equivalence of $(1)$ and $(2),(1-e) A$ is a maximal $\ell$-ideal, hence $Z_{\ell}(1-e)$ is a singleton, whose complement is $Z_{\ell}(e)$. This yields that $(1-e) A$ is an isolated point of $Y_{A}$. Conversely, if $N$ is an isolated point of $Y_{A}$, then $Y_{A} \backslash\{N\}$ is clopen. Since $\operatorname{Clop}\left(Y_{A}\right)$ is isomorphic to $\operatorname{Id}(A)$, there is $e \in \operatorname{Id}(A)$ such that $Y_{A} \backslash\{N\}=Z_{\ell}(e)$. But then $\{N\}=$ $Z_{\ell}(1-e)=\{(1-e) A\}$, so $e$ is a primitive idempotent by the equivalence of $(1)$ and (2). Thus, $X_{A}=\{(1-e) A \mid e \in \operatorname{Prim}(A)\}$.

Let $A \in \boldsymbol{b a} \boldsymbol{\ell}$. It follows from the proof of [5, Thm. 4.3] that $A \cong B(X)$ for some set $X$ iff $A \in \boldsymbol{d} \boldsymbol{b a} \boldsymbol{\ell}$ and $\operatorname{Id}(A)$ is atomic. For the reader's convenience we give a proof of this in Proposition 3.4, along with another equivalent condition that $A \cong B\left(X_{A}\right)$. For this we require the following definition.

Definition 3.3. For $A \in \boldsymbol{b a} \boldsymbol{\ell}$, define $\vartheta_{A}: A \rightarrow B\left(X_{A}\right)$ as the composition $\vartheta_{A}=\kappa_{A} \circ \zeta_{A}$ where $\zeta_{A}: A \rightarrow C\left(Y_{A}\right)$ is the Yosida representation and $\kappa_{A}: C\left(Y_{A}\right) \rightarrow B\left(X_{A}\right)$ sends $f \in C\left(Y_{A}\right)$ to its restriction to $X_{A}$. Since both $\zeta_{A}$ and $\kappa_{A}$ are morphisms in $\boldsymbol{b a} \boldsymbol{\ell}$, so is $\vartheta_{A}$.

For a set $X$ and $x \in X$ let

$$
N_{x}=\{f \in B(X) \mid f(x)=0\} .
$$

Note that if $X \in \mathrm{CReg}$, then $M_{x}=N_{x} \cap C^{*}(X)$.

Proposition 3.4. The following are equivalent for $A \in \mathbf{b a \ell}$.

(1) $A \in \boldsymbol{d} \boldsymbol{b} \boldsymbol{a} \boldsymbol{\ell}$ and $\operatorname{Id}(A)$ is atomic.

(2) There is a set $X$ such that $A \cong B(X)$.

(3) $\vartheta_{A}: A \rightarrow B\left(X_{A}\right)$ is an isomorphism.

Proof. $(3) \Rightarrow(1)$. We have $B\left(X_{A}\right) \in \boldsymbol{b a} \boldsymbol{\ell}$ and infinite joins and meets of bounded subsets of $B\left(X_{A}\right)$ are pointwise, hence exist in $B\left(X_{A}\right)$. Therefore, $B\left(X_{A}\right)$ is Dedekind complete, hence $B\left(X_{A}\right) \in \boldsymbol{d} \boldsymbol{b a} \boldsymbol{\ell}$. In addition, idempotents of $B\left(X_{A}\right)$ are exactly the characteristic functions of subsets of $X_{A}$, and primitive idempotents the characteristic functions of singletons. Thus, the boolean algebra of idempotents of $B\left(X_{A}\right)$ is isomorphic to the powerset $\wp\left(X_{A}\right)$. It follows that $\operatorname{Id}\left(B\left(X_{A}\right)\right)$ is atomic.

$(1) \Rightarrow(2)$. Since $A \in \boldsymbol{d} \boldsymbol{b a} \boldsymbol{\ell}, Y_{A}$ is extremally disconnected and $A \cong C\left(Y_{A}\right)$ by Corollary 2.6. So since $\operatorname{Id}(A)$ is atomic, $X_{A}$ is dense in $Y_{A}$. Therefore, $Y_{A}$ is homeomorphic to the StoneCech compactification $\beta\left(X_{A}\right)$ of the discrete space $X_{A}$ (see, e.g., [12, p. 96]). Thus, $C\left(Y_{A}\right) \cong$ $C\left(\beta\left(X_{A}\right)\right) \cong C^{*}\left(X_{A}\right)$. Since $X_{A}$ is discrete, $C^{*}\left(X_{A}\right)=B\left(X_{A}\right)$, yielding that $A \cong B\left(X_{A}\right)$. 
$(2) \Rightarrow(3)$. We may assume that $A=B(X)$. Then the primitive idempotents of $A$ are characteristic functions of points of $X$. We have $N_{x}=\left(1-\chi_{\{x\}}\right) A$, so $X_{A}=\left\{N_{x} \mid x \in X\right\}$ by Lemma 3.2. Let $f \in A$. Then $\vartheta_{A}(f)\left(N_{x}\right)=r$ iff $f-r \in N_{x}$ iff $f(x)=r$. From this it follows that $\vartheta_{A}$ is 1-1. To see that $\vartheta_{A}$ is onto, since $A$ is Dedekind complete, $\zeta_{A}: A \rightarrow C\left(Y_{A}\right)$ is an isomorphism. In addition, since $\operatorname{Id}(A)$ is atomic, as we already pointed out, $Y_{A}$ is homeomorphic to the Stone-Cech compactification of $X_{A}$. Therefore, $\kappa_{A}: C\left(Y_{A}\right) \rightarrow B\left(X_{A}\right)$ is onto. Thus, $\vartheta_{A}$ is onto, hence an isomorphism.

This proposition motivates the following definition.

Definition 3.5. We call $A \in \boldsymbol{b a} \boldsymbol{\ell}$ a basic algebra if $A$ is Dedekind complete and $\operatorname{Id}(A)$ is atomic.

Remark 3.6. It is shown in [5, Thm. 4.3] that for $A \in \mathbf{d b a \ell}$, the conditions of Proposition 3.4 are also equivalent to $A$ having essential socle.

We recall that a unital $\ell$-algebra homomorphism $\alpha: A \rightarrow B$ between $A, B \in \boldsymbol{d} \boldsymbol{b a} \boldsymbol{\ell}$ is normal if it preserves all existing joins (and hence all existing meets). Let balg be the category of basic algebras and normal $\ell$-algebra homomorphisms.

Convention 3.7. For a map $\varphi: X \rightarrow Y$, define $\varphi^{+}: B(Y) \rightarrow B(X)$ by $\varphi^{+}(f)=f \circ \varphi$.

Remark 3.8. If $X, Y \in \mathrm{CReg}$ and $\varphi: X \rightarrow Y$ is continuous, then $\varphi^{*}: C^{*}(Y) \rightarrow C^{*}(X)$ is the restriction of $\varphi^{+}: B(Y) \rightarrow B(X)$.

It is easy to see that $\varphi^{+}$is a normal $\ell$-algebra homomorphism. Thus, we have a contravariant functor $B:$ Set $\rightarrow$ balg which associates with each set $X$ the basic algebra $B(X)$, and with each map $\varphi: X \rightarrow Y$ the normal $\ell$-algebra homomorphism $\varphi^{+}: B(Y) \rightarrow B(X)$.

The contravariant functor $X: \boldsymbol{b a l g} \rightarrow$ Set is defined as follows. With each basic algebra $A$ we associate the set $X_{A}$ of isolated points of $Y_{A}$. To define the action of the functor on morphisms, we recall that a continuous map $\varphi: X \rightarrow Y$ is skeletal if $F$ nowhere dense in $Y$ implies that $\varphi^{-1}(F)$ is nowhere dense in $X$, and that $\varphi$ is quasi-open if $U$ nonempty open in $X$ implies that the interior of $\varphi(U)$ is nonempty in $Y$. It is well known that the two concepts of skeletal and quasi-open maps coincide in KHaus. Let $\alpha: A \rightarrow B$ be a normal $\ell$-algebra homomorphism. By [4, Thm. 7.6], $\alpha_{\star}: Y_{B} \rightarrow Y_{A}$ is skeletal. Therefore, it is quasi-open, and hence $\alpha_{*}$ sends isolated points of $Y_{B}$ to isolated points of $Y_{A}$. Thus, the restriction of $\alpha_{*}$ to $X_{B}$ is a well-defined map from $X_{B}$ to $X_{A}$.

Convention 3.9. Let $\alpha_{+}: X_{B} \rightarrow X_{A}$ be the restriction of $\alpha_{\star}: Y_{B} \rightarrow Y_{A}$.

Consequently, we have a contravariant functor $X: \boldsymbol{b a l g} \rightarrow$ Set which associates with each $A \in \boldsymbol{b a l g}$ the set $X_{A}$ and with each normal $\ell$-algebra homomorphism $\alpha: A \rightarrow B$ the map $\alpha_{+}: X_{B} \rightarrow X_{A}$.

Theorem 3.10. The categories Set and balg are dually equivalent, and the dual equivalence is established by the functors $B$ and $X$. 
Proof. We define a natural transformation $\eta$ from the identity functor on Set to $X B$ by $\eta_{X}(x)=N_{x}$. Given a function $\varphi: X \rightarrow X^{\prime}$, we have the following diagram.

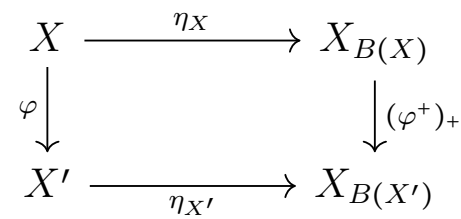

To see that the diagram is commutative, let $x \in X$. Then $\eta_{X^{\prime}}(\varphi(x))=N_{\varphi(x)}$. Also,

$$
\begin{aligned}
\left(\varphi^{+}\right)_{+}\left(\eta_{X}(x)\right) & =\left(\varphi^{+}\right)_{+}\left(N_{x}\right)=\left(\varphi^{+}\right)^{-1}\left(N_{x}\right)=\left\{f \in B\left(X^{\prime}\right) \mid \varphi^{+}(f) \in N_{x}\right\} \\
& =\left\{f \in B\left(X^{\prime}\right) \mid f(\varphi(x))=0\right\}=N_{\varphi(x)} .
\end{aligned}
$$

Thus, $\eta_{X^{\prime}} \circ \varphi=\left(\varphi^{+}\right)_{+} \circ \eta_{X}$, and hence $\eta$ is a natural transformation. Since $\eta_{X}$ is a bijection, we conclude that $\eta$ is a natural isomorphism.

We show that $\vartheta$ is a natural transformation from the identity functor on balg to $B X$. Given a normal homomorphism $\alpha: A \rightarrow A^{\prime}$, we have the following diagram.

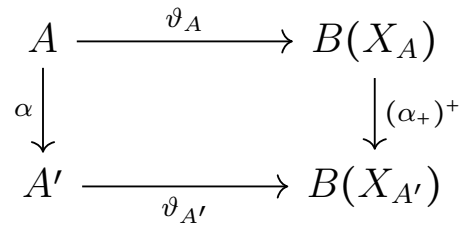

The diagram factors into the larger diagram.

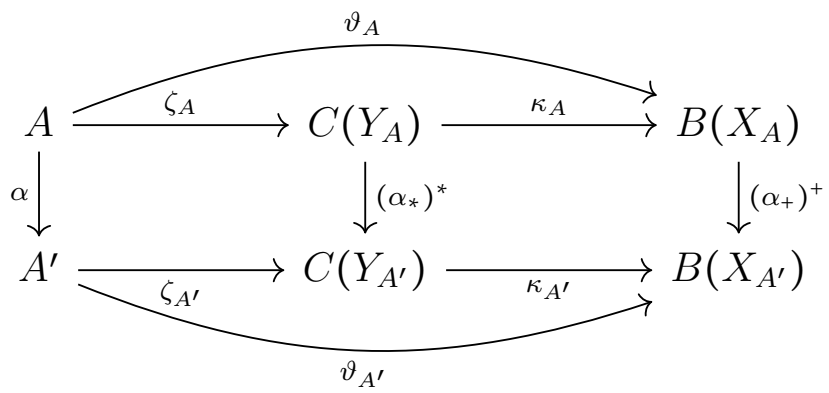

We have $\left(\alpha_{*}\right)^{*} \circ \zeta_{A}=\zeta_{A^{\prime}} \circ \alpha$ by Gelfand-Naimark-Stone duality, so the left square commutes. To see that the right square commutes, let $f \in C\left(Y_{A}\right)$. Then

$$
\left(\alpha_{+}\right)^{+}\left(\kappa_{A}(f)\right)=\left(\alpha_{+}\right)^{+}\left(\left.f\right|_{X_{A}}\right)=\left(\left.f\right|_{X_{A}}\right) \circ \alpha_{+} .
$$

On the other hand,

$$
\left(\kappa_{A^{\prime}} \circ\left(\alpha_{*}\right)^{*}\right)(f)=\kappa_{A^{\prime}}\left(f \circ \alpha_{\star}\right)=\left.\left(f \circ \alpha_{*}\right)\right|_{X_{A^{\prime}}} .
$$

For $M \in X_{A^{\prime}}$, we have

$$
\left(\left.f\right|_{X_{A}} \circ \alpha_{+}\right)(M)=f\left(\alpha_{*}(M)\right)=\left.\left(f \circ \alpha_{*}\right)\right|_{X_{A}}(M),
$$

so $\left.f\right|_{X_{A}} \circ \alpha_{+}=\left.\left(f \circ \alpha_{*}\right)\right|_{X_{A^{\prime}}}$, and hence the right square commutes. Therefore, $\left(\alpha_{+}\right)^{+} \circ \vartheta_{A}=$ $\vartheta_{A^{\prime}} \circ \alpha$, and so $\vartheta$ is a natural transformation. It is a natural isomorphism by Proposition 3.4 . Thus, $B$ and $X$ yield a dual equivalence of Set and $\boldsymbol{b a l g}$. 
Remark 3.11. The duality of Theorem 3.10 relates to Tarski duality as follows. Define a covariant functor Id : balg $\rightarrow$ CABA by sending each $A \in \boldsymbol{b a l g}$ to the boolean algebra $\operatorname{Id}(A)$ and a normal homomorphism $\alpha: A \rightarrow B$ to its restriction to $\operatorname{Id}(A)$. It is easy to see that $\operatorname{Id}$ is well defined, and we have the following diagram.

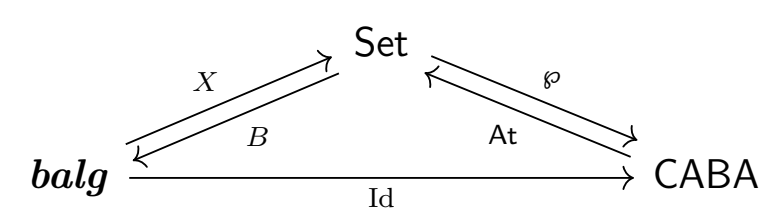

The functor $\wp:$ Set $\rightarrow$ CABA is the composition $\operatorname{Id} \circ B$, and the composition $B \circ$ At $:$ CABA $\rightarrow$ balg takes $C \in \mathrm{CABA}$ and sends it to $B(X)$, where $X$ is the set of isolated points of the Stone space of $C$, so $B \circ \mathrm{At} \cong \mathrm{Id}$.

\section{Compactifications And Basic extensions}

We recall (see, e.g., [10, Sec. 3.5]) that a compactification of a completely regular space $X$ is a pair $(Y, e)$, where $Y$ is a compact Hausdorff space and $e: X \rightarrow Y$ is a topological embedding such that the image $e[X]$ is dense in $Y$. Suppose that $e: X \rightarrow Y$ and $e^{\prime}: X \rightarrow Y^{\prime}$ are compactifications. As usual, we write $e \leq e^{\prime}$ provided there is a continuous map $f: Y^{\prime} \rightarrow Y$ with $f \circ e^{\prime}=e$.

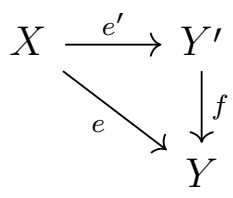

The relation $\leq$ is reflexive and transitive. Two compactifications $e$ and $e^{\prime}$ are said to be equivalent if $e \leq e^{\prime}$ and $e^{\prime} \leq e$. It is well known that $e$ and $e^{\prime}$ are equivalent iff there is a homeomorphism $f: Y^{\prime} \rightarrow Y$ with $f \circ e^{\prime}=e$. The equivalence classes of compactifications of $X$ form a poset whose largest element is the Stone-Čech compactification $s: X \rightarrow \beta X$. There are many constructions of $\beta X$.

Convention 4.1. We will follow Stone [17] in viewing $\beta X$ as the maximal ideals of $C^{*}(X)$. Since maximal ideals of $C^{*}(X)$ are the same as maximal $\ell$-ideals (see [13, Lem. 1.1] or [2, Prop. 4.2]), throughout this paper we identify $\beta X$ with $Y_{C^{*}(X)}$, and hence the embedding $s: X \rightarrow \beta X$ sends $x$ to $M_{x}=\left\{f \in C^{*}(X) \mid f(x)=0\right\}$.

In the classical setting, one considers compactifications of a fixed base space $X$. The following category of compactifications, without a fixed base space, was studied in [6].

Definition 4.2. Let Comp be the category whose objects are compactifications $e: X \rightarrow Y$ and whose morphisms are pairs $(f, g)$ of continuous maps such that the following diagram commutes.

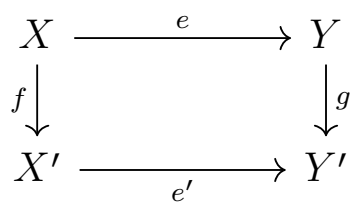


The composition of two morphisms $\left(f_{1}, g_{1}\right)$ and $\left(f_{2}, g_{2}\right)$ is defined to be $\left(f_{2} \circ f_{1}, g_{2} \circ g_{1}\right)$.

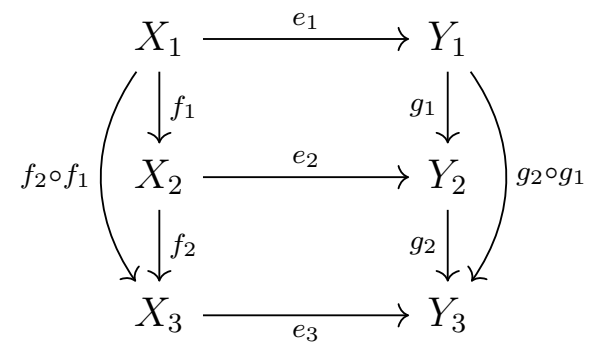

It is straightforward to see that a morphism $(f, g)$ in Comp is an isomorphism iff both $f$ and $g$ are homeomorphisms.

Convention 4.3. For a compactification $e: X \rightarrow Y$ let $e^{b}: C(Y) \rightarrow B(X)$ be given by $e^{b}(f)=f \circ e$.

Remark 4.4. We have that $e^{b}=\iota \circ e^{*}$ where $\iota: C^{*}(X) \rightarrow B(X)$ is the inclusion map.

Proposition 4.5. If $e: X \rightarrow Y$ is a compactification, then $e^{b}: C(Y) \rightarrow B(X)$ is a monomorphism in $\boldsymbol{b a} \boldsymbol{\ell}$, and each element of $B(X)$ is a join of meets of elements from $e^{b}[C(Y)]$.

Proof. That the $\ell$-algebra homomorphism $e^{b}: C(Y) \rightarrow B(X)$ is 1-1 follows from the fact that the image of $e$ is dense in $Y$. Next we show that every primitive idempotent in $B(X)$ is a meet of elements from $e^{b}[C(Y)]$. Let $b \in \operatorname{Prim}(B(X))$. Then $b$ is the characteristic function of a singleton set, so $b=\chi_{\{x\}}$ for some $x \in X$. Let

$$
S=\left\{e^{b}(g) \mid 0 \leq g \in C(Y) \text { and } g(e(x))=1\right\} .
$$

We claim $\chi_{\{x\}}=\wedge S$. It is clear that $\chi_{\{x\}} \leq \wedge S$. Suppose by way of contradiction that $\chi_{\{x\}} \neq \wedge S$. Then there exist $x^{\prime} \in X$ and $\varepsilon>0$ such that $x \neq x^{\prime}$ and $g\left(e\left(x^{\prime}\right)\right)>\varepsilon$ for all $0 \leq g \in C(Y)$ with $g(e(x))=1$. Since $e$ is $1-1$, we have $e(x) \neq e\left(x^{\prime}\right)$, and since $Y$ is completely regular, there exists $0 \leq g \in C(Y)$ with $g(e(x))=1$ and $g\left(e\left(x^{\prime}\right)\right)=0$. This contradiction shows that $\chi_{\{x\}}=\wedge S$. Therefore, every primitive idempotent in $B(X)$ is a meet of elements from $e^{b}[C(Y)]$.

Let $0 \leq c \in B(X)$. By Lemma 3.2, for each $b \in \operatorname{Prim}(B(X))$ there is $r_{b} \in \mathbb{R}$ such that $c b=r_{b} b$. Since $\operatorname{Id}(B(X))$ is atomic, we have $1=\bigvee \operatorname{Prim}(B(X))$, and so since $c \geq 0$,

$$
\begin{aligned}
c & =c \cdot 1=c \cdot \bigvee\{b \in \operatorname{Prim}(B(X))\} \\
& =\bigvee\{c b \mid b \in \operatorname{Prim}(B(X))\} \\
& =\bigvee\left\{r_{b} b \mid b \in \operatorname{Prim}(B(X))\right\} .
\end{aligned}
$$

Since $c \geq 0$, each $r_{b} \geq 0$, and as the primitive idempotent $b$ is a meet of elements from $e^{b}[C(Y)]$, so is $r_{b} b$. This yields that every positive element of $B(X)$ is a join of meets of elements from $e^{b}[C(Y)]$.

To finish the argument, let $c \in B(X)$. Then there is $s \in \mathbb{R}$ with $c+s \geq 0$. By the previous argument, we may then write $c+s=\bigvee\left\{r_{b} b \mid b \in \operatorname{Prim}(B(X))\right\}$ for some $0 \leq r_{b} \in \mathbb{R}$. Therefore,

$$
c=(c+s)-s=\bigvee\left\{r_{b} b-s \mid b \in \operatorname{Prim}(B(X))\right\} .
$$


Since $r_{b} b$ is a meet of elements from $e^{b}[C(Y)]$ and $s$ is a scalar, $r_{b} b-s$ is also a meet of elements from $e^{b}[C(Y)]$, Thus, every element of $B(X)$ is a join of meets of elements from $e^{b}[C(Y)]$.

Definition 4.6. Let $\alpha: A \rightarrow B$ be a monomorphism in $\boldsymbol{b a} \boldsymbol{\ell}$.

(1) We say $\alpha[A]$ is join-meet dense in $B$ if each element of $B$ is a join of meets from $\alpha[A]$.

(2) We say $\alpha[A]$ is meet-join dense in $B$ if each element of $B$ is a meet of joins from $\alpha[A]$.

Remark 4.7. Let $\alpha: A \rightarrow B$ be a monomorphism in $\boldsymbol{b a} \boldsymbol{\ell}$ with $B$ a basic algebra.

(1) $\alpha[A]$ is join-meet dense in $B$ iff each primitive idempotent of $B$ is a meet from $\alpha[A]$. The right-to-left implication follows from the proof of Proposition 4.5. For the leftto-right implication, let $b \in \operatorname{Prim}(B)$. Then $b$ is a join of meets of elements from $\alpha[A]$, so there is a meet $c$ of elements from $\alpha[A]$ with $0<c$ and $c \leq b$. Because $0 \leq 1-b$, we have $0 \leq c(1-b) \leq b(1-b)=0$. Therefore, $c(1-b)=0$, so $c=c b$. By Lemma 3.2, $c b=r b$ for some nonzero scalar $r$. Thus, $r>0$. This implies $b=r^{-1} c$ is a meet of elements from $\alpha[A]$.

(2) $\alpha[A]$ is join-meet dense in $B$ iff $\alpha[A]$ is meet-join dense in $B$. For the left-to-right implication, let $b \in B$. We may write $-b=\bigvee\left\{\wedge\left\{\alpha\left(a_{i j}\right) \mid j \in J\right\} \mid i \in I\right\}$ for some $a_{i j} \in A$. Then $b=\bigwedge\left\{\bigvee\left\{\alpha\left(-a_{i j}\right) \mid j \in J\right\} \mid i \in I\right\}$, which shows $b$ is a meet of joins from $\alpha[A]$. The reverse implication is similar.

Proposition 4.5 motivates the following key definition of the article.

Definition 4.8. Let $A \in \boldsymbol{b a} \boldsymbol{\ell}, B \in \boldsymbol{b a l g}$, and $\alpha: A \rightarrow B$ be a monomorphism in $\boldsymbol{b a} \boldsymbol{\ell}$. We call $\alpha: A \rightarrow B$ a basic extension if $\alpha[A]$ is join-meet dense in $B$.

\section{Example 4.9.}

(1) If $e: X \rightarrow Y$ is a compactification, then $e^{b}: C(Y) \rightarrow B(X)$ is a basic extension by Proposition 4.5.

(2) If $X$ is completely regular, then the inclusion map $\iota: C^{*}(X) \rightarrow B(X)$ is a basic extension. To see this, let $s: X \rightarrow \beta X$ be the Stone-Čech compactification of $X$. By $(1), s^{b}$ is a basic extension. Since $s^{b}$ is an isomorphism from $C(\beta X)$ to $C^{\star}(X)$, we see that $\iota$ is a basic extension. In fact, $s^{b}$ is isomorphic to $\iota$ in the category of basic extensions described in Definition 4.10.

(3) If $Y \in$ KHaus, it follows from (1) that the inclusion map $\iota: C(Y) \rightarrow B(Y)$ is a basic extension.

(4) If $A \in \boldsymbol{b a} \boldsymbol{\ell}$, it follows from [5, Thm. 2.9] that $\vartheta_{A}: A \rightarrow B\left(Y_{A}\right)$ is a basic extension.

\section{Definition 4.10.}


(1) Let basic be the category whose objects are basic extensions and whose morphisms are pairs $(\rho, \sigma)$ of morphisms in $\boldsymbol{b a} \boldsymbol{\ell}$ with $\sigma$ normal and $\sigma \circ \alpha=\alpha^{\prime} \circ \rho$.

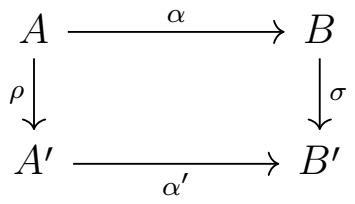

The composition of two morphisms $\left(\rho_{1}, \sigma_{1}\right)$ and $\left(\rho_{2}, \sigma_{2}\right)$ is defined to be $\left(\rho_{2} \circ \rho_{1}, \sigma_{2} \circ\right.$ $\left.\sigma_{1}\right)$.

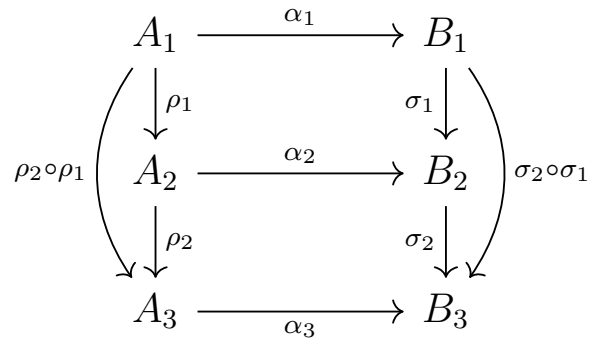

(2) Let ubasic be the full subcategory of basic consisting of the basic extensions $\alpha$ : $A \rightarrow B$ where $A \in \boldsymbol{u b a \ell}$.

Theorem 4.11. ubasic is a reflective subcategory of basic.

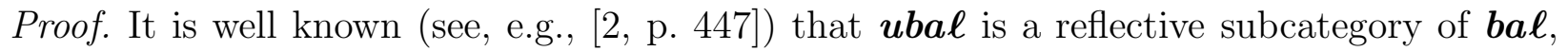
and the reflector sends $A \in \boldsymbol{b a} \boldsymbol{\ell}$ to $C\left(Y_{A}\right)$. If $\alpha: A \rightarrow C$ is a morphism in $\boldsymbol{b a} \boldsymbol{\ell}$ with $C \in \boldsymbol{u b a} \boldsymbol{\ell}$, let $\widehat{\alpha}: C\left(Y_{A}\right) \rightarrow C$ be the unique morphism in $\boldsymbol{b a} \boldsymbol{\ell}$ with $\widehat{\alpha} \circ \zeta_{A}=\alpha$.

Define a functor $r:$ basic $\rightarrow$ ubasic as follows. If $\alpha: A \rightarrow B$ is a basic extension, then $\widehat{\alpha}\left[C\left(Y_{A}\right)\right]$ is join-meet dense in $B$ because it contains $\alpha[A]$. Thus, $\widehat{\alpha}: C\left(Y_{A}\right) \rightarrow B$ is a basic extension. We set $\mathrm{r}(\alpha)=\widehat{\alpha}$. If $(\rho, \sigma)$ is a morphism in basic, we set $\mathrm{r}(\rho, \sigma)=\left(\left(\rho_{\star}\right)^{*}, \sigma\right)$. To see that $\left(\left(\rho_{*}\right)^{*}, \sigma\right)$ is a morphism in basic, consider the following diagram.

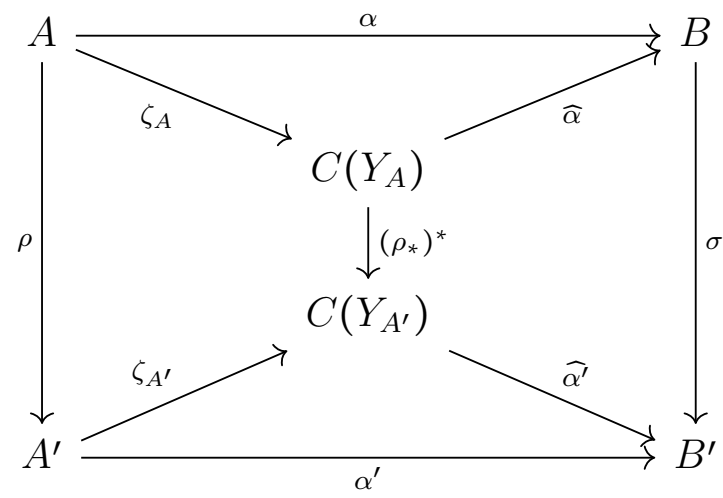

We have

$$
\sigma \circ \widehat{\alpha} \circ \zeta_{A}=\sigma \circ \alpha=\alpha^{\prime} \circ \rho=\widehat{\alpha^{\prime}} \circ \zeta_{A^{\prime}} \circ \rho=\widehat{\alpha^{\prime}} \circ\left(\rho_{*}\right)^{*} \circ \zeta_{A} .
$$

Since $\zeta_{A}$ is epic (see, e.g., [2, Lem. 2.9]), $\sigma \circ \widehat{\alpha}=\widehat{\alpha^{\prime}} \circ\left(\rho_{\star}\right)^{*}$. Therefore, $\left(\left(\rho_{\star}\right)^{*}, \sigma\right)$ is a morphism in basic. Since $C$ is a functor, it is straightforward to see that $r$ is a functor. For a basic 
extension $\alpha: A \rightarrow B$, we let $\mathrm{r}_{\alpha}$ be the morphism $\left(\zeta_{A}, 1_{B}\right)$ from $\alpha$ to $\widehat{\alpha}$, where $1_{B}$ is the identity on $B$. Suppose that $(\rho, \sigma)$ is a morphism to an object $\alpha^{\prime}: A^{\prime} \rightarrow B^{\prime}$ of $\boldsymbol{u b a s i c}$, so $A^{\prime} \in \boldsymbol{u b a \boldsymbol { l } \text { . }}$

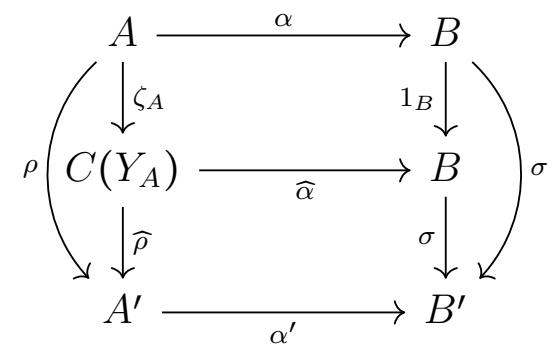

Then $(\widehat{\rho}, \sigma)$ is a morphism in $\boldsymbol{u b a s i c}$, and since $\widehat{\rho}$ is the unique morphism satisfying $\widehat{\rho} \circ \zeta_{A}=\rho$, it follows that $(\widehat{\rho}, \sigma)$ is the unique morphism satisfying $(\widehat{\rho}, \sigma) \circ\left(\zeta_{A}, 1_{B}\right)=(\rho, \sigma)$. This proves that $\boldsymbol{u b a s i c}$ is a reflective subcategory of basic.

Define a contravariant functor $\mathrm{E}:$ Comp $\rightarrow$ basic as follows. If $e: X \rightarrow Y$ is a compactification, define $\mathrm{E}(\mathrm{e})$ to be the basic extension $e^{b}: C(Y) \rightarrow B(X)$. For a morphism $(f, g)$ in Comp

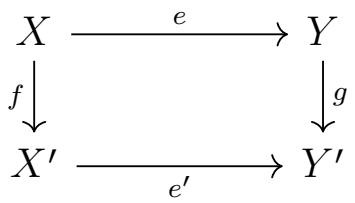

define $\mathrm{E}(f, g)$ to be the pair $\left(g^{*}, f^{+}\right)$

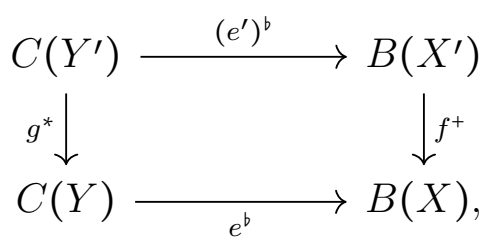

Proposition 4.12. E : Comp $\rightarrow$ basic is a contravariant functor such that each object of ubasic is isomorphic to $\mathrm{E}(e)$ for some compactification $e: X \rightarrow Y$.

Proof. Let $e: X \rightarrow Y$ be a compactification. By Proposition 4.5, $e^{b}: C(Y) \rightarrow B(X)$ is a basic extension. Thus, $\mathrm{E}(e) \in$ basic. Let $(f, g)$ be a morphism in Comp. Then $\mathrm{E}(f, g)=\left(g^{*}, f^{+}\right)$. We show that $\mathrm{E}(f, g)$ is a morphism in basic. Let $a \in C\left(Y^{\prime}\right)$. Then

$$
\begin{aligned}
\left(f^{+} \circ\left(e^{\prime}\right)^{b}\right)(a) & =f^{+}\left(\left(e^{\prime}\right)^{b}(a)\right)=\left(a \circ e^{\prime}\right) \circ f=a \circ\left(e^{\prime} \circ f\right)=a \circ(g \circ e) \\
& =(a \circ g) \circ e=e^{b}\left(g^{*}(a)\right)=\left(e^{b} \circ g^{*}\right)(a) .
\end{aligned}
$$

This yields $f^{+} \circ\left(e^{\prime}\right)^{b}=e^{b} \circ g^{*}$. Because $g^{*}, f^{+}$are morphisms in $\boldsymbol{b a} \boldsymbol{\ell}$ and $f^{+}$is normal, $\mathrm{E}(f, g)$ is a morphism in basic. From the definition of composition in Comp and basic it is elementary to see that $\mathrm{E}$ preserves composition and identity morphisms. Thus, $\mathrm{E}$ is a contravariant functor. That each object of ubasic is isomorphic to $\mathrm{E}(e)$ for some compactification $e: X \rightarrow Y$ follows from the definition of ubasic and Gelfand-NaimarkStone duality. 


\section{The FunCtor C : basic $\rightarrow$ Comp}

Convention 5.1. For a morphism $\alpha: A \rightarrow B$ in $\boldsymbol{b a} \boldsymbol{\ell}$, let $\alpha_{b}: X_{B} \rightarrow Y_{A}$ be the restriction of $\alpha_{\star}: Y_{B} \rightarrow Y_{A}$ to $X_{B}$.

Definition 5.2. Let $\alpha: A \rightarrow B$ be a monomorphism in $\boldsymbol{b a} \boldsymbol{\ell}$ with $B$ a basic algebra. Define a topology $\tau_{\alpha}$ on $X_{B}$ as the least topology making $\alpha_{b}: X_{B} \rightarrow Y_{A}$ continuous.

Remark 5.3. Following usual terminology, we will refer to the topological space $\left(X_{B}, \tau_{\alpha}\right)$ as $X_{B}$ when there is no danger of confusion about which topology we are using.

Proposition 5.4. Let $\alpha: A \rightarrow B$ be a monomorphism in bal with $B$ a basic algebra. Then $\alpha_{b}: X_{B} \rightarrow Y_{A}$ is 1-1 iff $\alpha[A]$ is join-meet dense in $B$.

Proof. By Remark 4.7(1), it is sufficient to show that $\alpha_{b}$ is $1-1$ iff each $b \in \operatorname{Prim}(B)$ is meet of elements from $\alpha[A]$.

First suppose that $\alpha_{b}$ is $1-1$. Let $b \in \operatorname{Prim}(B)$. We show that $1-b$ is a join of elements from $\alpha[A]$. Let $g$ be the join of all $\alpha(a) \in A$ with $0 \leq \alpha(a) \leq 1-b$. Clearly $g \leq 1-b$. To see that $1-b \leq g$, let $c \in \operatorname{Prim}(B)$ with $c \neq b$. Since $\alpha_{b}$ is $1-1$, there is $\alpha(a) \in(1-b) B$ with $\alpha(a) \notin(1-c) B$. By replacing $a$ by $|a|$, and then multiplying by an appropriate scalar, we may assume $0 \leq a \leq 1$. Since $\alpha(a) \in(1-b) B$ and $1-b$ is an idempotent, $\alpha(a)=(1-b) \alpha(a)$, so $\alpha(a) b=0$. Also $\alpha(a) \notin(1-c) B$ implies $\alpha(a)(1-c) \neq \alpha(a)$, so $\alpha(a) c \neq 0$. Since $a>0$, Lemma 3.2 yields $\alpha(a) c=r c$ for some real number $r>0$. We have $c=r^{-1} r c=r^{-1} \alpha(a) c \leq$ $r^{-1} \alpha(a)$ since $c \leq 1$. Let $a^{\prime}=1 \wedge r^{-1} a$. Then $a^{\prime} \in A$ and $c \leq \alpha\left(a^{\prime}\right)$. Moreover, $0 \leq a^{\prime} \leq r^{-1} a$, so $0 \leq \alpha\left(a^{\prime}\right) b \leq r^{-1} \alpha(a) b=0$. This implies $\alpha\left(a^{\prime}\right) b=0$, so $\alpha\left(a^{\prime}\right)(1-b)=\alpha\left(a^{\prime}\right)$. Since $\alpha\left(a^{\prime}\right) \leq 1$, we have $\alpha\left(a^{\prime}\right)(1-b) \leq 1-b$, hence $\alpha\left(a^{\prime}\right) \leq 1-b$. Therefore, $\alpha\left(a^{\prime}\right) \leq g$ by the definition of $g$. This yields $c \leq \alpha\left(a^{\prime}\right) \leq g$ for each $c \neq b$. Since $1-b$ is the join of all primitive idempotents $c \neq b$, we get $1-b \leq g \leq 1-b$, so $1-b=g$. This shows that $1-b$ is a join of elements from $\alpha[A]$, and hence $b$ is a meet of elements from $\alpha[A]$.

Conversely, suppose that each $b \in \operatorname{Prim}(B)$ is a meet of elements from $\alpha[A]$. Let $M, N \in X_{B}$ and $\alpha^{-1}(M)=\alpha^{-1}(N)$. By Lemma 3.2, there are $b, c \in \operatorname{Prim}(B)$ with $M=(1-b) B$ and $N=(1-c) B$. Thus, $\alpha^{-1}((1-b) B)=\alpha^{-1}((1-c) B)$. As $b$ is primitive, $1-b$ is the join of the elements from $\alpha[A]$ below it. Because $0 \leq 1-b$, these elements can be taken to be positive. Take $a \in A$ with $0 \leq \alpha(a) \leq 1-b$. By the calculation in the first paragraph above, $\alpha(a) b=0$, and so $\alpha(a)=\alpha(a)(1-b)$. Therefore, $a \in \alpha^{-1}((1-b) B)=\alpha^{-1}((1-c) B)$, and hence $\alpha(a) \in(1-c) B$. Since $1-c$ is an idempotent, it follows that $\alpha(a)=\alpha(a)(1-c)$, which implies $\alpha(a) \leq 1-c$. As $1-b$ is the join of all such $\alpha(a)$ we see that $1-b \leq 1-c$. Because $b, c$ are primitive idempotents, this implies $b=c$, and so $M=N$. Thus, $\alpha_{b}$ is $1-1$.

Theorem 5.5. If $\alpha: A \rightarrow B$ is a basic extension, then $X_{B}$ is completely regular and $\alpha_{b}$ : $X_{B} \rightarrow Y_{A}$ is a compactification.

Proof. By Proposition [5.4, $\alpha_{b}: X_{B} \rightarrow Y_{A}$ is 1-1. Therefore, $X_{B}$ is homeomorphic to $\alpha_{b}\left[X_{B}\right]$ by Definition 5.2, and so $X_{B}$ is completely regular. Since $\alpha$ is 1-1, $\alpha_{\star}: Y_{B} \rightarrow Y_{A}$ is onto, and $X_{B}$ is dense in $Y_{B}$ because $B$ is atomic. Thus, $\alpha_{b}\left[X_{B}\right]$ is dense in $Y_{A}$, and hence $\alpha_{b}: X_{B} \rightarrow Y_{A}$ is a compactification. 
Example 5.6. Let $X$ be a completely regular space. By Example 4.9(2), the inclusion $\iota: C^{*}(X) \rightarrow B(X)$ is a basic extension. Then $\iota_{b}: X_{B(X)} \rightarrow Y_{C^{*}(X)}$ is a compactification by Theorem 5.5. We claim that $\iota_{b}$ is isomorphic to the Stone-Čech compactification $s: X \rightarrow \beta X$ in Comp. By Convention 4.1, $\beta X=Y_{C^{*}(X)}$ and $s(x)=M_{x}$. Consider the following diagram

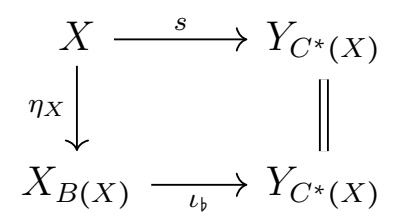

where we recall from the proof of Theorem 3.10 that $\eta_{X}: X \rightarrow X_{B(X)}$, sending $x$ to $N_{x}$, is a bijection. The diagram commutes because

$$
\iota_{b}\left(\eta_{X}(x)\right)=\iota^{-1}\left(N_{x}\right)=\left\{f \in C^{*}(X) \mid f(x)=0\right\}=M_{x}=s(x) .
$$

To see that $\eta_{X}$ is a homeomorphism, since $\left(X_{B(X)}, \tau_{\iota}\right)$ is completely regular, it has a basis of cozero sets. Let $U$ be a cozero set in $X_{B(X)}$. Then there is $f \in C^{*}(X)$ with $U=\{M \in$ $\left.X_{B(X)} \mid f \notin M\right\}$. We have

$$
\eta_{X}^{-1}(U)=\left\{x \in X \mid N_{x} \in U\right\}=\left\{x \in X \mid f \notin N_{x}\right\}=\{x \in X \mid f(x) \neq 0\},
$$

which is the cozero set of $f$ in $X$. Since $\eta_{X}$ is a bijection, we conclude that $\eta_{X}$ is a homeomorphism. Thus, $\iota_{b}$ and $s$ are isomorphic in Comp.

Lemma 5.7. Suppose that $e: X \rightarrow Y$ and $e^{\prime}: X^{\prime} \rightarrow Y^{\prime}$ are compactifications, $g: Y \rightarrow Y^{\prime}$ is a continuous map, and $f: X \rightarrow X^{\prime}$ is a map such that $e^{\prime} \circ f=g \circ e$. Then $f$ is continuous.

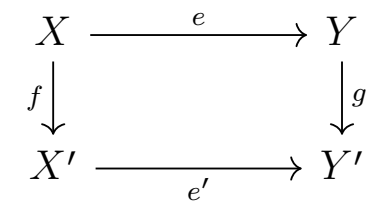

Proof. Let $U$ be open in $X^{\prime}$. Then there is an open set $V$ of $Y^{\prime}$ with $U=\left(e^{\prime}\right)^{-1}(V)$. We have

$$
f^{-1}(U)=f^{-1}\left(\left(e^{\prime}\right)^{-1}(V)\right)=\left(e^{\prime} \circ f\right)^{-1}(V)=(g \circ e)^{-1}(V)=e^{-1}\left(g^{-1}(V)\right),
$$

so $f^{-1}(U)$ is open in $X$. Thus, $f$ is continuous.

Define a functor $\mathrm{C}:$ basic $\rightarrow$ Comp as follows. If $\alpha: A \rightarrow B$ is a basic extension, set $\mathrm{C}(\alpha)$ to be the compactification $\alpha_{b}: X_{B} \rightarrow Y_{A}$. For a morphism $(\rho, \sigma)$ in basic

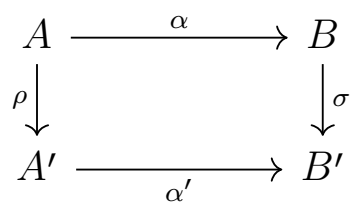

define $\mathrm{C}(\rho, \sigma)$ to be $\left(\sigma_{+}, \rho_{*}\right)$, where $\sigma_{+}$is the restriction of $\sigma_{*}: Y_{B^{\prime}} \rightarrow Y_{B}$ to $X_{B^{\prime}}$.

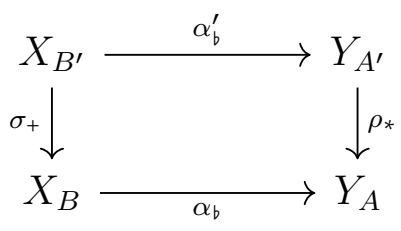


Since $\sigma \circ \alpha=\alpha^{\prime} \circ \rho$ we have $\rho_{\star} \circ \alpha_{*}^{\prime}=\alpha_{\star} \circ \sigma_{*}$. Restricting both sides to $X_{B^{\prime}}$ shows this diagram is commutative. As an immediate consequence of Lemma 5.7, we have:

Lemma 5.8. $\sigma_{+}$is continuous, and hence $\left(\sigma_{+}, \rho_{\star}\right)$ is a morphism in Comp.

Proposition 5.9. C : basic $\rightarrow$ Comp is a contravariant functor.

Proof. Let $\alpha: A \rightarrow B$ be a basic extension. By Theorem 5.5, $\alpha_{b}: X_{B} \rightarrow Y_{A}$ is a compactification. Therefore, $\mathrm{C}(\alpha) \in$ Comp. By Lemma 5.8, if $(\rho, \sigma)$ is a morphism in basic, then $\mathrm{C}(\rho, \sigma)=\left(\sigma_{+}, \rho_{*}\right)$ is a morphism in Comp. Suppose that $\left(\rho_{1}, \sigma_{1}\right)$ and $\left(\rho_{2}, \sigma_{2}\right)$ are composable morphisms in basic. Then

$$
\mathrm{C}\left(\left(\rho_{2}, \sigma_{2}\right) \circ\left(\rho_{1}, \sigma_{1}\right)\right)=\mathrm{C}\left(\rho_{2} \circ \rho_{1}, \sigma_{2} \circ \sigma_{1}\right)=\left(\left(\sigma_{2} \circ \sigma_{1}\right)_{+},\left(\rho_{2} \circ \rho_{1}\right)_{*}\right) .
$$

Since $\left(\rho_{2} \circ \rho_{1}\right)_{*}=\left(\rho_{1}\right)_{*} \circ\left(\rho_{2}\right)_{*}$ and $\left(\sigma_{2} \circ \sigma_{1}\right)_{+}=\left(\sigma_{1}\right)_{+} \circ\left(\sigma_{2}\right)_{+}$, we see that

$$
\begin{aligned}
\mathrm{C}\left(\left(\rho_{2}, \sigma_{2}\right) \circ\left(\rho_{1}, \sigma_{1}\right)\right) & =\left(\left(\sigma_{1}\right)_{+} \circ\left(\sigma_{2}\right)_{+},\left(\rho_{1}\right)_{*} \circ\left(\rho_{2}\right)_{*}\right) \\
& =\left(\left(\sigma_{1}\right)_{+},\left(\rho_{1}\right)_{*}\right) \circ\left(\left(\sigma_{2}\right)_{+},\left(\rho_{2}\right)_{*}\right)=\mathrm{C}\left(\rho_{1}, \sigma_{1}\right) \circ \mathrm{C}\left(\rho_{2}, \sigma_{2}\right),
\end{aligned}
$$

which shows that $\mathrm{C}$ preserves composition. It is clear that $\mathrm{C}$ preserves identity morphisms. Thus, $\mathrm{C}$ is a contravariant functor.

\section{Duality Between Comp And ubasic}

In this section we prove that the functors $E$ and $C$ yield a dual adjunction between Comp and basic, which restricts to a dual equivalence between Comp and ubasic. For this we require the following two lemmas.

Lemma 6.1. Let $\alpha: A \rightarrow B$ be a basic extension. Then $\left(\zeta_{A}, \vartheta_{B}\right)$ is a morphism in basic, and it is an isomorphism provided $A \in \boldsymbol{u b a \ell}$.

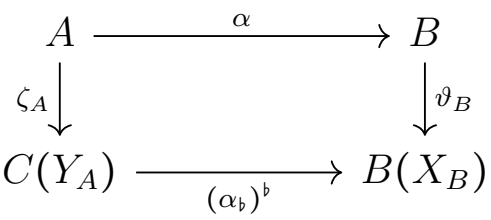

Proof. The map $\vartheta_{B}$ is an isomorphism by Proposition 3.4, and so it is a normal homomorphism. To see that $\left(\zeta_{A}, \vartheta_{B}\right)$ is a morphism in basic we need to show $\left(\alpha_{b}\right)^{b} \circ \zeta_{A}=\vartheta_{B} \circ \alpha$. We have the following diagram.

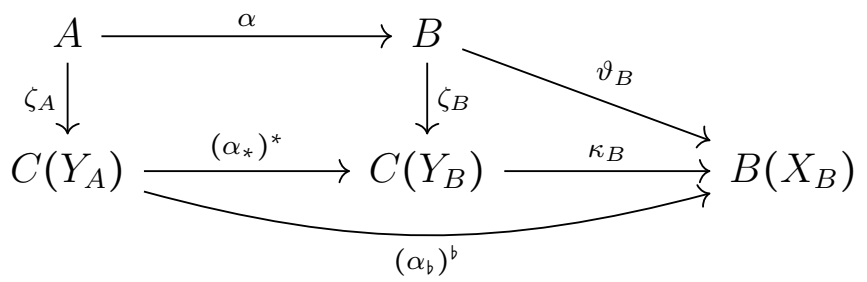

The left-hand side commutes by Gelfand-Naimark-Stone duality, and the right-hand side commutes by the definition of $\vartheta_{B}$. Therefore, $\left(\alpha_{b}\right)^{b} \circ \zeta_{A}=\vartheta_{B} \circ \alpha$, and hence $\left(\zeta_{A}, \vartheta_{B}\right)$ is a morphism in basic. If $A \in \boldsymbol{u b a} \boldsymbol{\ell}$, then $\zeta_{A}$ is an isomorphism. Since $\vartheta_{B}$ is an isomorphism, $\left(\zeta_{A}, \vartheta_{B}\right)$ is an isomorphism. 
Lemma 6.2. Let $e: X \rightarrow Y$ be a compactification. Then $\eta_{X}: X \rightarrow X_{B(X)}$ is a homeomorphism, and $\left(\eta_{X}, \xi_{Y}\right)$ is an isomorphism of compactifications between e and $\left(e^{b}\right)_{b}$.

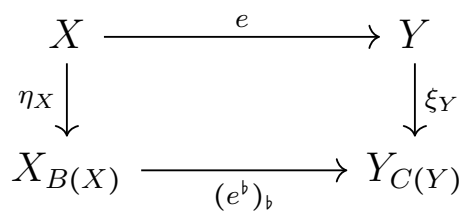

Proof. We already observed in the proof of Theorem 3.10 that $\eta_{X}$ is a bijection. We show that $\xi_{Y} \circ e=\left(e^{b}\right)_{b} \circ \eta_{X}$. Let $x \in X$. Then $\xi_{Y}(e(x))=M_{e(x)}$. We have

$$
\left(e^{b}\right)_{b}\left(\eta_{X}(x)\right)=\left\{c \in C(Y) \mid e^{b}(c)(x)=0\right\}=\{c \in C(Y) \mid c(e(x))=0\}=M_{e(x)} .
$$

Therefore, $\left(e^{b}\right)_{b} \circ \eta_{X}=\xi_{Y} \circ e$. Then, by Lemma 5.7, $\eta_{X}$ is continuous, and so $\left(\eta_{X}, \xi_{Y}\right)$ is a morphism in Comp. Since $\eta_{X}$ is a bijection and $\xi_{Y}$ is a homeomorphism, applying Lemma 5.7 (with the pair $\eta_{X}^{-1}$ and $\left.\xi_{Y}^{-1}\right)$, shows that $\eta_{X}$ is a homeomorphism. Thus, $\left(\eta_{X}, \xi_{Y}\right)$ is an isomorphism in Comp.

Theorem 6.3. The functors $\mathrm{E}: \mathrm{Comp} \rightarrow$ basic and $\mathrm{C}:$ basic $\rightarrow$ Comp define a dual adjunction of categories that restricts to a dual equivalence between Comp and ubasic.

Proof. Propositions 4.12 and 5.9 show that E and C are contravariant functors. We first show that CE is naturally isomorphic to the identity functor on Comp. The functor $\mathrm{E}$ sends $e: X \rightarrow Y$ to $e^{b}: C(Y) \rightarrow B(X)$. Then $C$ sends this to $\left(e^{b}\right)_{b}: X_{B(X)} \rightarrow Y_{C(Y)}$. By Lemma6.2, $\left(\eta_{X}, \xi_{Y}\right)$ is an isomorphism in Comp.

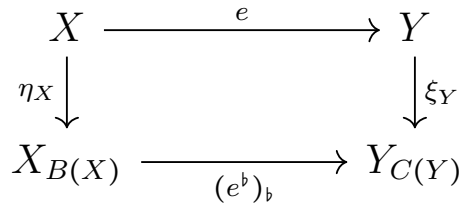

Let $(f, g)$ be be a morphism in Comp. Then $\mathrm{E}(f, g)=\left(g^{*}, f^{+}\right)$, and so $\mathrm{CE}(f, g)=$ $\mathrm{C}\left(g^{*}, f^{+}\right)=\left(\left(f^{+}\right)_{+},\left(g^{*}\right)_{*}\right)$. Thus, $\left(\left(f^{+}\right)_{+},\left(g^{*}\right)_{*}\right)$ is a morphism in Comp.

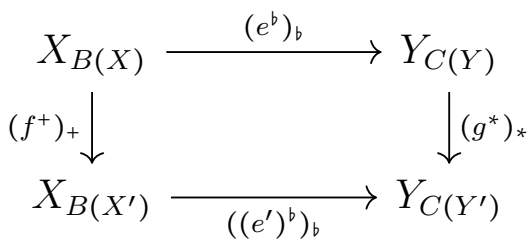

We define a natural transformation $p$ from the identity functor on Comp to CE as follows. For a compactification $e: X \rightarrow Y$ we set $p_{e}=\left(\eta_{X}, \xi_{Y}\right)$. By Lemma 6.2, $p_{e}$ is an isomorphism 
in Comp. We have the following diagram.

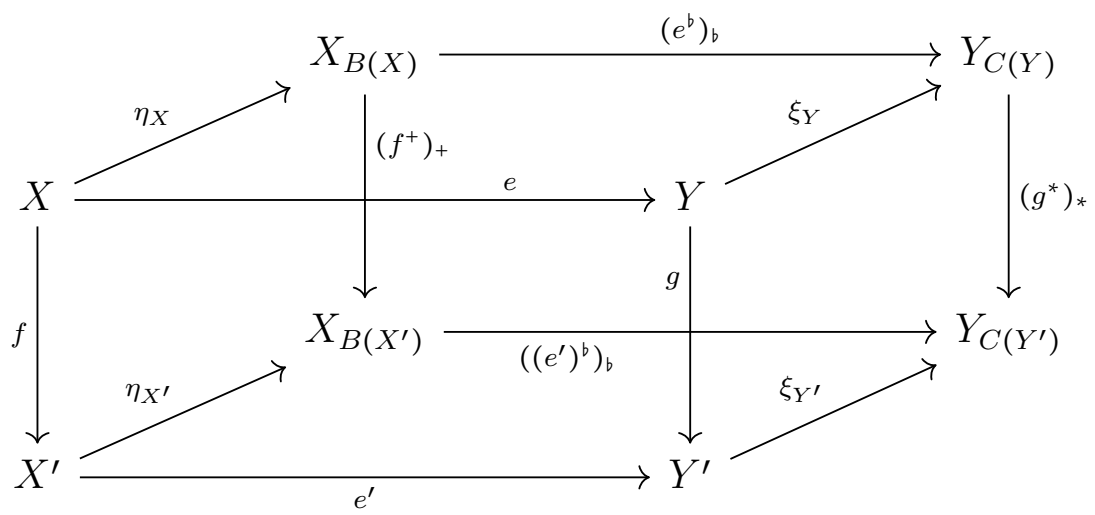

The front and back faces of this cube are commutative because $(f, g)$ and $\operatorname{CE}(f, g)=$ $\left(\left(f^{+}\right)_{+},\left(g^{*}\right)_{*}\right)$ are morphisms in Comp. The top and bottom faces are commutative since $p_{e}$ and $p_{e^{\prime}}$ are morphisms in Comp. The right face is commutative by Gelfand-Naimark-Stone duality, and the left face is commutative by Theorem 3.10. The commutativity of this cube shows that $p$ is a natural transformation. In fact, since $p_{e}$ is an isomorphism in Comp, we see that $p$ is a natural isomorphism.

We next define a natural transformation $q$ from the identity functor on basic to EC. Given a basic extension $\alpha: A \rightarrow B$, the functor $\mathrm{C}$ sends it to $\alpha_{b}: X_{B} \rightarrow Y_{A}$. This is then sent by $\mathrm{E}$ to $\left(\alpha_{b}\right)^{\mathrm{b}}: C\left(Y_{A}\right) \rightarrow B\left(X_{B}\right)$.

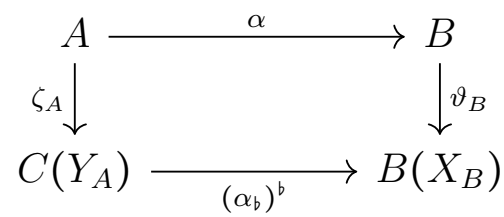

The pair $\left(\zeta_{A}, \vartheta_{B}\right)$ is a morphism in basic by Lemma 6.1. Define $q$ by setting $q_{\alpha}=\left(\zeta_{A}, \vartheta_{B}\right)$ for a basic extension $\alpha: A \rightarrow B$. By Lemma 6.1, $q_{\alpha}$ is a morphism in basic. To show naturality, let $(\rho, \sigma)$ be a morphism in basic. We have the following diagram.

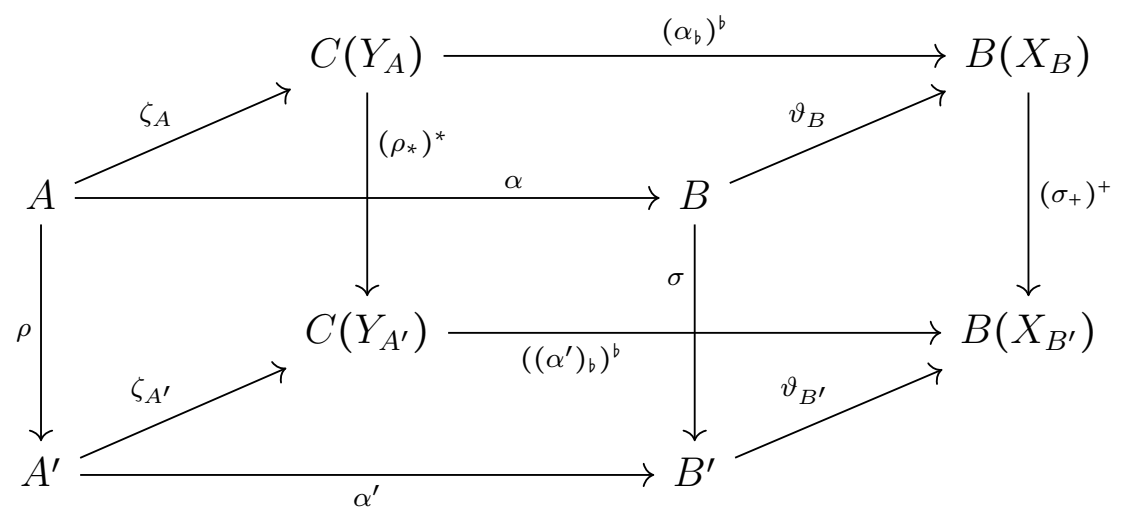

The front and back faces of this cube are commutative because $(\rho, \sigma)$ and $\operatorname{EC}(\rho, \sigma)=$ $\left(\left(\rho_{*}\right)^{*},\left(\sigma_{+}\right)^{+}\right)$are morphisms in basic. The top and bottom faces are commutative because $q_{\alpha}$ and $q_{\alpha^{\prime}}$ are morphisms in basic. The left face is commutative by Gelfand-Naimark-Stone duality, and the right face is commutative by Theorem 3.10. This shows that $q$ is a natural 
transformation. In addition, if $\alpha: A \rightarrow B$ is an object of $\boldsymbol{u b a s i c}$, then $q_{\alpha}$ is an isomorphism by Lemma 6.1. Therefore, $C$ and $E$ yield a dual equivalence between Comp and ubasic. This together with Theorem 4.11 gives that $\mathrm{E}$ and $\mathrm{C}$ define a dual adjunction between Comp and basic.

We conclude this section by relating Theorem 6.3 to Gelfand-Naimark-Stone duality.

Remark 6.4. Let I : KHaus $\rightarrow$ Comp be the functor sending a compact Hausdorff space $Y$ to the compactification $1_{Y}: Y \rightarrow Y$ where $1_{Y}$ is the identity map. Let also $\mathrm{G}: \boldsymbol{b} \boldsymbol{a} \boldsymbol{\ell} \rightarrow \boldsymbol{b a s i c}$ be the functor sending $A \in \boldsymbol{b} \boldsymbol{a} \boldsymbol{\ell}$ to the basic extension $\vartheta_{A}: A \rightarrow B\left(Y_{A}\right)$ (see Example 4.9(3)). We have the following diagram.

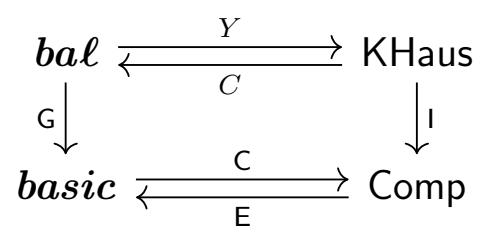

Let $A \in \boldsymbol{b a} \boldsymbol{\ell}$. Then $\mathrm{I}(Y(A))$ is the compactification $Y_{A} \rightarrow Y_{A}$. On the other hand, $\mathrm{C}(\mathrm{G}(A))$ is the image under $\mathrm{C}$ of the basic extension $A \rightarrow B\left(Y_{A}\right)$, which is $X_{B\left(Y_{A}\right)} \rightarrow Y_{A}$, and this is naturally isomorphic to $Y_{A} \rightarrow Y_{A}$. Therefore, $\mathrm{I} \circ Y$ and $\mathrm{C} \circ \mathrm{G}$ are naturally isomorphic.

Next, let $Y \in$ KHaus. Then $\mathrm{G}(C(Y))$ is the basic extension $C(Y) \rightarrow B\left(Y_{C(Y)}\right)$. Also, $\mathrm{E}(\mathrm{I}(Y))$ is the image under $\mathrm{E}$ of the compactification $Y \rightarrow Y$, which is the extension $C(Y) \rightarrow B(Y)$. Since $Y$ and $Y_{C(Y)}$ are naturally homeomorphic, $\mathrm{G} \circ C$ and $\mathrm{E} \circ \mathrm{I}$ are naturally isomorphic. Consequently, the duality of Theorem 6.3 extends Gelfand-Naimark-Stone duality.

\section{Duality for COMPletely REgular SPACES}

In this final section we show how to use Theorem 6.3 to derive duality for the category CReg of completely regular spaces. For this we will need to introduce the concept of a maximal basic extension and connect it to the Stone-Čech compactification.

Since the Stone-Cech compactification is the largest among compactifications of a given completely regular space, it is natural to define a maximal basic extension as the largest with respect to the corresponding order among basic extensions $\alpha: A \rightarrow B$ that yield the same completely regular topology on $X_{B}$. We call such basic extensions compatible. To give a purely algebraic description of compatibility requires some preparation.

Let $e: X \rightarrow Y$ and $e^{\prime}: X \rightarrow Y^{\prime}$ be two compactifications of the same completely regular space $X$. Then we have two basic extensions $e^{b}: C(Y) \rightarrow B(X)$ and $\left(e^{\prime}\right)^{b}: C\left(Y^{\prime}\right) \rightarrow B(X)$. While the images of $C(Y)$ and $C\left(Y^{\prime}\right)$ in $B(X)$ are in general different, as we will see shortly, they have isomorphic Dedekind completions. For this we need to recall Dilworth's notion of a normal lower semicontinuous function [9].

Let $X$ be completely regular. For $x \in X$ let $\mathcal{N}_{x}$ be the family of open neighborhoods of $x$. For $f \in B(X)$ set

$$
f^{\star}(x)=\inf _{U \in \mathcal{N}_{x}} \sup _{y \in U} f(y) \quad \text { and } \quad f_{*}(x)=\sup _{U \in \mathcal{N}_{x}} \inf _{y \in U} f(y)
$$


We recall that $f$ is lower semicontinuous if $f=f_{*}$, upper semicontinuous if $f=f^{*}$, and normal (lower semicontinuous) if $\left(f^{*}\right)_{*}=f$. We set $N(X)=\left\{f \in B(X) \mid\left(f^{*}\right)_{*}=f\right\}$.

\section{Remark 7.1.}

(1) Dilworth [9] showed that if we view $C^{*}(X)$ and $N(X)$ as lattices, then $N(X)$ is the Dedekind completion of $C^{*}(X)$.

(2) Dăneţ [8] showed that Dilworth's result generalizes to the setting of vector lattices, and hence $N(X)$ is the Dedekind completion of $C^{*}(X)$ as a vector lattice.

(3) Clearly $C^{*}(X) \in \boldsymbol{b a} \boldsymbol{\ell}$. It follows from [4, Ex. 8.4(2)] that there is a multiplication on $N(X)$ extending the multiplication on $C^{*}(X)$ such that $N(X) \in \boldsymbol{b a} \boldsymbol{\ell}$. Consequently, $N(X)$ is the Dedekind completion of $C^{*}(X)$ also as an $\ell$-algebra 1

Lemma 7.2. Let $X$ be a subspace of a topological space $Y$. If $f$ is a bounded lower (resp. upper) semicontinuous real-valued function on $X$, then there is a bounded lower (resp. upper) semicontinuous real-valued function $g$ on $Y$ with $\left.g\right|_{X}=f$.

Proof. Let $f$ be a bounded lower semicontinuous function on $X$. Then $s:=\sup \{f(x) \mid x \in X\}$ exists. We extend $f$ to a function $f^{\prime}$ on $Y$ by setting $f^{\prime}(y)=s$ for all $y \in Y \backslash X$. Then $f^{\prime}$ is a bounded function on $Y$. We define $g$ on $Y$ by setting

$$
g(y)=\sup _{U \in \mathcal{N}_{y}} \inf _{z \in U} f^{\prime}(z)
$$

for each $y \in Y$. Then $g$ is lower semicontinuous by [9, Sec. 3]. Let $x \in X$. By the definition of $f^{\prime}$, if $U \in \mathcal{N}_{x}$, then $\inf \left\{f^{\prime}(y) \mid y \in U\right\}=\inf \{f(z) \mid z \in U \cap X\}$. Therefore,

$$
g(x)=\sup _{U \in \mathcal{N}_{x}} \inf _{y \in U} f^{\prime}(y)=\sup _{U \in \mathcal{N}_{x}} \inf _{z \in U \cap X} f(z) .
$$

Because $X$ is a subspace of $Y$, we see that $\left\{U \cap X \mid U \in \mathcal{N}_{x}\right\}$ is the collection of open neighborhoods of $x$ in $X$. Thus, since $f$ is lower semicontinuous on $X$,

$$
f(x)=\sup _{U \in \mathcal{N}_{x}} \inf _{z \in U \cap X} f(z)=g(x)
$$

for each $x \in X$, and hence $\left.g\right|_{X}=f$. The argument for upper semicontinuous functions is similar and left to the reader.

Lemma 7.3. Let $\alpha: A \rightarrow B$ be a basic extension and let $f \in C^{*}\left(X_{B}\right)$. Then $f$ is a pointwise join and meet of elements from $\alpha_{b}^{*} \zeta_{A}[A]$.

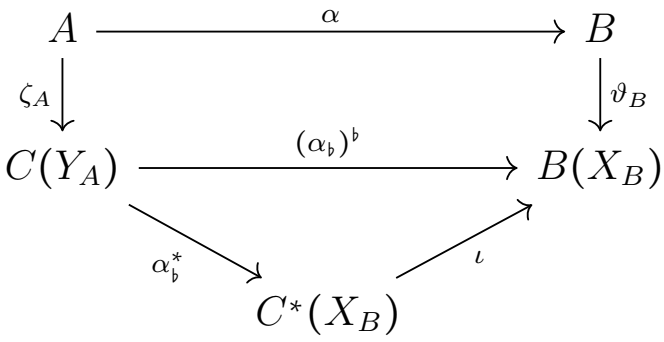

\footnotetext{
${ }^{1}$ We point out that neither the lattice nor the algebra operations on $N(X)$ are pointwise.
} 
Proof. Since $f$ is continuous, by Lemma 7.2 there is a lower semicontinuous function $g \epsilon$ $B\left(Y_{A}\right)$ and an upper semicontinuous function $h \in B\left(Y_{A}\right)$ with $g \circ \alpha_{b}=f=h \circ \alpha_{b}$. By [9, Lem. 4.1] (and its dual for lower semicontinuous functions), $g=\bigvee S$ is a pointwise join of elements from $C\left(Y_{A}\right)$ and $h$ is a pointwise meet of elements from $C\left(Y_{A}\right)$. Because the map $\left(\alpha_{\mathrm{b}}\right)^{+}: B\left(Y_{A}\right) \rightarrow B\left(X_{B}\right)$ sending $f$ to $f \circ \alpha_{\mathrm{b}}$ is a normal homomorphism,

$$
f=g \circ \alpha_{b}=\alpha_{b}^{+}(g)=\bigvee\left\{\alpha_{b}^{+}(k) \mid k \in S\right\}=\bigvee\left\{\alpha_{b}^{*}(k) \mid k \in S\right\}
$$

is a join of elements from $\left(\alpha_{b}\right)^{*}\left[C\left(Y_{A}\right)\right]$. Similarly, $f$ is a meet of elements from $\alpha_{b}^{*}\left[C\left(Y_{A}\right)\right]$. Thus, $f$ is both a pointwise join and meet from $\alpha_{b}^{*}\left[C\left(Y_{A}\right)\right]$. The argument of [5, Lem. 2.8] shows that each element of $C\left(Y_{A}\right)$ is both a pointwise join and meet from $\zeta_{A}[A]$. Each $k \in S$ then can be written, in $B\left(Y_{A}\right)$, as $k=\bigvee\left\{\zeta_{A}(a) \mid a \in T_{k}\right\}$ for some $T_{k} \subseteq A$. Set $T=\bigcup\left\{T_{k} \mid k \in S\right\}$. We have

$$
\begin{aligned}
f & \left.\left.=\bigvee\left\{\alpha_{b}^{*}(k) \mid k \in S\right\}=\bigvee\left\{\alpha_{b}^{*}\left(\bigvee \zeta_{A}(a)\right) \mid a \in T_{k}\right\}\right) \mid k \in S\right\} \\
& =\bigvee\left\{\alpha_{b}^{*}\left(\zeta_{A}(a)\right) \mid a \in T\right\} .
\end{aligned}
$$

Therefore, $f$ is a pointwise join from $\alpha_{b}^{*} \zeta_{A}[A]$. Repeating the argument above but replacing $g$ by $h$ and joins with meets shows that $f$ is a pointwise meet from $\alpha_{b}^{*} \zeta_{A}[A]$.

As an immediate consequence of Lemma 7.3 we obtain:

Lemma 7.4. Let $e: X \rightarrow Y$ be a compactification. Then each $f \in C^{*}(X)$ is a pointwise join and meet from $e^{*}[C(Y)]$.

Lemma 7.5. If $e: X \rightarrow Y$ is a compactification, then $N(X)$ is isomorphic to $N(Y)$ in $\boldsymbol{b a} \boldsymbol{\ell}$.

Proof. It follows from Lemma 7.4 that $e^{*}[C(Y)]$ is join-dense and meet-dense in $C^{*}(X)$. Consequently, $C(Y)$ and $C^{\star}(X)$ have isomorphic Dedekind completions. Thus, by Remark 7.1 (3), $N(Y)$ and $N(X)$ are isomorphic in $\boldsymbol{b} \boldsymbol{a} \boldsymbol{\ell}$.

Remark 7.6. In fact, the restriction of $e^{+}: B(Y) \rightarrow B(X)$ to $N(Y)$ is a well-defined isomorphism of $N(Y)$ and $N(X)$. Since we do not require this fact in what follows, we omit the proof.

Let $e: X \rightarrow Y$ be a compactification and $\alpha:=e^{b}: C(Y) \rightarrow B(X)$ the corresponding basic extension. Using [9, Lem. 4.1] as motivation, we define $u_{\alpha}, l_{\alpha}: B(X) \rightarrow B(X)$ as follows. For $f \in B(X)$ let

$$
u_{\alpha}(f)=\bigwedge\{\alpha(g) \mid g \in C(Y), f \leq \alpha(g)\} \quad \text { and } \quad l_{\alpha}(f)=\bigvee\{\alpha(g) \mid g \in C(Y), \alpha(g) \leq f\} .
$$

We set

$$
N_{\alpha}(X)=\left\{f \in B(X) \mid l_{\alpha} u_{\alpha}(f)=f\right\} .
$$

Lemma 7.7. Let $e: X \rightarrow Y$ be a compactification and $\alpha=e^{b}: C(Y) \rightarrow B(X)$ the corresponding basic extension. Then $N_{\alpha}(X)=N(X)$.

Proof. Let $f \in B(X)$. By [9, Lem. 4.1], $f^{*}=\wedge\left\{g \in C^{*}(X) \mid f \leq g\right\}$. By Lemma 7.4, each $g \in C^{*}(X)$ is a pointwise meet from $\alpha[C(Y)]$. Thus, $f^{*}=u_{\alpha}(f)$. A similar argument yields that $f_{*}=l_{\alpha}(f)$. Thus, $\left(f^{*}\right)_{*}=l_{\alpha} u_{\alpha}(f)$. From this and the definitions of $N_{\alpha}(X)$ and $N(X)$ it follows that $N_{\alpha}(X)=N(X)$. 
This motivates the following definition.

Definition 7.8. Let $\alpha: A \rightarrow B$ be a basic extension.

(1) For $b \in B$ set

$$
u_{\alpha}(b)=\bigwedge\{\alpha(a) \mid a \in A, b \leq \alpha(a)\} \quad \text { and } \quad l_{\alpha}(b)=\bigvee\{\alpha(a) \mid a \in A, \alpha(a) \leq b\} .
$$

(2) Let $N_{\alpha}=\left\{b \in B \mid l_{\alpha} u_{\alpha}(b)=b\right\}$.

We are ready to define when two basic basic extensions are compatible.

Definition 7.9. We call two basic extensions $\alpha: A \rightarrow B$ and $\gamma: C \rightarrow B$ compatible if $N_{\alpha}=N_{\gamma}$.

\section{Lemma 7.10.}

(1) If $\alpha: A \rightarrow B$ is a basic extension, then $\vartheta_{B}\left(N_{\alpha}\right)=N\left(X_{B}\right)$.

(2) Two basic extensions $\alpha: A \rightarrow B$ and $\gamma: C \rightarrow B$ are compatible iff $\tau_{\alpha}=\tau_{\gamma}$.

Proof. (1). By Lemma 7.7, it suffices to show that $\vartheta_{B}\left(N_{\alpha}\right)=N_{\alpha}\left(X_{B}\right)$. We first show that $\vartheta_{B}\left(u_{\alpha}(b)\right)=u_{\alpha}\left(\vartheta_{B}(b)\right)$ for each $b \in B$. To see this, since $\vartheta_{B}$ is an isomorphism and $\vartheta_{B} \alpha(a)=\alpha_{b}^{*} \zeta_{A}(a)$,

$$
\vartheta_{B}\left(u_{\alpha}(b)\right)=\bigwedge\left\{\vartheta_{B}(\alpha(a)) \mid a \in A, b \leq \alpha(a)\right\}=\bigwedge\left\{\alpha_{b}^{*} \zeta_{A}(a) \mid a \in A, b \leq \alpha(a)\right\} .
$$

On the other hand, $u_{\alpha}\left(\vartheta_{B}(b)\right)=\bigwedge\left\{\alpha_{b}^{*}(g) \mid g \in C\left(Y_{A}\right), \vartheta_{B}(b) \leq g\right\}$. By [5, Lem. 2.8], each $g \in C\left(Y_{A}\right)$ is a pointwise meet from $\zeta_{A}[A]$. Consequently,

$$
u_{\alpha}\left(\vartheta_{B}(b)\right)=\bigwedge\left\{\alpha_{b}^{*} \zeta_{A}(a) \mid a \in A, \vartheta_{B}(b) \leq \alpha_{b}^{*} \zeta_{A}(a)\right\} .
$$

Since $\vartheta_{B} \alpha(a)=\alpha_{b}^{*} \zeta_{A}(a)$ and $\vartheta_{B}$ is an isomorphism, $b \leq \alpha(a)$ iff $\vartheta_{B}(b) \leq \alpha_{b}^{*} \zeta_{A}(a)$. Thus, $\vartheta_{B}\left(u_{\alpha}(b)\right)=u_{\alpha}\left(\vartheta_{B}(b)\right)$. Similarly, $\vartheta_{B}\left(l_{\alpha}(b)\right)=l_{\alpha}\left(\vartheta_{B}(b)\right)$. From this it follows that $\vartheta_{B}\left(N_{\alpha}\right)=$ $N_{\alpha}\left(X_{B}\right)$.

(2). Because we are working with two topologies, to avoid confusion, we write $N\left(X_{B}, \tau_{\alpha}\right)$ and $N\left(X_{B}, \tau_{\gamma}\right)$. First suppose that $\tau_{\alpha}=\tau_{\gamma}$. Then $N\left(X_{B}, \tau_{\alpha}\right)=N\left(X_{B}, \tau_{\gamma}\right)$. Therefore, by (1), $\vartheta_{B}\left(N_{\alpha}\right)=\vartheta_{B}\left(N_{\gamma}\right)$. Since $\vartheta_{B}$ is $1-1$, we conclude that $N_{\alpha}=N_{\gamma}$.

Conversely, suppose that $N_{\alpha}=N_{\gamma}$. Then $\vartheta_{B}\left(N_{\alpha}\right)=\vartheta_{B}\left(N_{\gamma}\right)$, and so $N\left(X_{B}, \tau_{\alpha}\right)=N\left(X_{B}, \tau_{\gamma}\right)$ by (1). To show that $\tau_{\alpha}=\tau_{\gamma}$, it suffices to show that $U \subseteq X_{B}$ is regular open in $\tau_{\alpha}$ iff it is regular open in $\tau_{\gamma}$. Now, $U$ is regular open in $\tau_{\alpha}$ iff the characteristic function $\chi_{U} \in N\left(X_{B}, \tau_{\alpha}\right)$ (see, e.g., [4, Ex. 4.11]). The corresponding statement for $\tau_{\gamma}$ holds for the same reason. Since $N\left(X_{B}, \tau_{\alpha}\right)=N\left(X_{B}, \tau_{\gamma}\right)$, we see that $U$ is regular open in $\tau_{\alpha}$ iff $U$ is regular open in $\tau_{\gamma}$. Thus, $\tau_{\alpha}=\tau_{\gamma}$.

We are now ready to define the notion of a maximal basic extension.

\section{Definition 7.11.}

(1) A basic extension $\alpha: A \rightarrow B$ is maximal provided that for every compatible extension $\gamma: C \rightarrow B$, there is a morphism $\delta: C \rightarrow A$ in $\boldsymbol{b a} \boldsymbol{\ell}$ such that $\alpha \circ \delta=\gamma$.

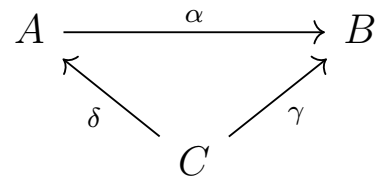


(2) Let $\boldsymbol{m b a s i c}$ be the full subcategory of basic consisting of maximal basic extensions.

We next give different characterizations of maximal basic extensions. Let $\alpha: A \rightarrow B$ be a basic extension. Then $\alpha_{b}: X_{B} \rightarrow Y_{A}$ is a continuous map, and so we have a morphism $\left(\alpha_{b}\right)^{*}: C\left(Y_{A}\right) \rightarrow C^{*}\left(X_{B}\right)$ in $\boldsymbol{b a} \boldsymbol{\ell}$.

Definition 7.12. Define $\mu: A \rightarrow C^{*}\left(X_{B}\right)$ as the composition $\mu=\left(\alpha_{b}\right)^{*} \circ \zeta_{A}$.

Since both $\zeta_{A}$ and $\left(\alpha_{b}\right)^{*}$ are morphisms in $\boldsymbol{b} \boldsymbol{a} \boldsymbol{\ell}$, so is $\mu$. In fact, $\mu$ is a monomorphism in $\boldsymbol{b a} \boldsymbol{\ell}$. To see this, note that $\zeta_{A}$ is $1-1$. We show that $\left(\alpha_{b}\right)^{*}$ is $1-1$. If $\left(\alpha_{b}\right)^{*}(f)=0$ for $f \in C\left(Y_{A}\right)$, then $f \circ \alpha_{b}=0$. Therefore, $\left.f\right|_{\alpha_{b}\left[X_{B}\right]}=0$. Since $\alpha_{b}\left[X_{B}\right]$ is dense in $Y_{A}$, we have $f=0$. Thus, $\left(\alpha_{b}\right)^{*}$ is $1-1$, and so $\mu$ is $1-1$.

Let $\iota: C^{*}\left(X_{B}\right) \rightarrow B\left(X_{B}\right)$ be the inclusion morphism. The following diagram commutes because the top half commutes by Gelfand-Naimark-Stone duality and the bottom half commutes by application of the relevant definitions.

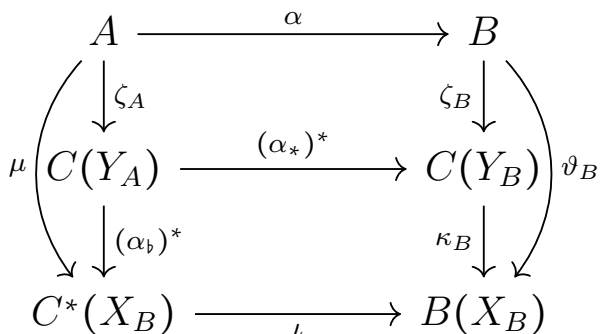

Proposition 7.13. The following are equivalent for a basic extension $\alpha: A \rightarrow B$.

(1) $\alpha$ is maximal.

(2) $\mu=\left(\alpha_{b}\right)^{*} \circ \zeta_{A}: A \rightarrow C^{*}\left(X_{B}\right)$ is an isomorphism.

(3) $A$ is uniformly complete and $\alpha_{b}: X_{B} \rightarrow Y_{A}$ is isomorphic to the Stone-Čech compactification $s: X_{B} \rightarrow \beta X_{B}$.

(4) $A$ is uniformly complete and $\alpha_{b}: X_{B} \rightarrow Y_{A}$ is equivalent to $s$.

(5) The only elements of $B$ that are both a join and meet of elements from $\alpha[A]$ are those that are in $\alpha[A]$.

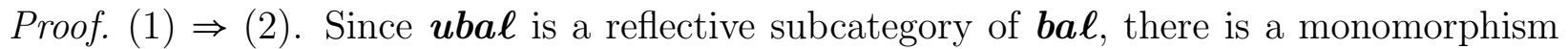
$\widehat{\alpha}: C\left(Y_{A}\right) \rightarrow B$ in $\boldsymbol{b a} \boldsymbol{\ell}$ with $\widehat{\alpha} \circ \zeta_{A}=\alpha$. As we pointed out in the proof of Theorem 4.11, $\widehat{\alpha}: C\left(Y_{A}\right) \rightarrow B$ is a basic extension. Since $\vartheta_{B}$ is an isomorphism (see Proposition 3.4), we may define $\gamma=\vartheta_{B}^{-1} \circ \iota$. By Example 4.9 $(2), \iota$ is a basic extension. Thus, $\gamma$ is a basic extension. By Example 5.6, $\tau_{\gamma}$ is equal to $\tau_{\alpha}$, and so $\gamma$ is compatible with $\alpha$. By (1), there is a morphism $\delta: C^{*}\left(X_{B}\right) \rightarrow A$ in $\boldsymbol{b a} \ell$ such that $\alpha \circ \delta=\gamma$.

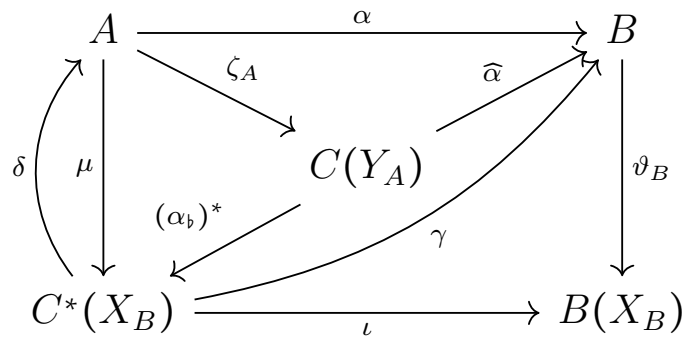


As we pointed out before the proposition, $\vartheta_{B} \circ \alpha=\iota \circ \mu$. Therefore,

$$
\iota \circ \mu \circ \delta=\vartheta_{B} \circ \alpha \circ \delta=\vartheta_{B} \circ \gamma=\iota .
$$

Since $\iota$ is monic, $\mu \circ \delta$ is the identity on $C^{*}\left(X_{B}\right)$. This implies $\mu$ is onto. Because $\mu$ is $1-1$, we conclude that $\mu$ is an isomorphism.

$(2) \Rightarrow(3)$. In light of $(2)$, it is clear that $A$ is uniformly complete. Let $f \in C\left(Y_{A}\right)$. Since the diagram above is commutative, $\left(\mu, \vartheta_{B}\right)$ is a morphism in basic. Because both $\mu$ and $\vartheta_{B}$ are isomorphisms, $\left(\mu, \vartheta_{B}\right)$ is an isomorphism in basic. Applying $C$ yields $\alpha_{b}$ and $\iota_{b}$ are isomorphic in Comp. Therefore, by Example 5.6, $\alpha_{b}$ and $s$ are isomorphic in Comp.

$(3) \Rightarrow(4)$. It is proved in [6, Thm. 3.3] that if a compactification $e: X \rightarrow Y$ is isomorphic to the Stone-Čech compactification $s: X \rightarrow \beta X$, then $e$ is equivalent to $s$.

$(4) \Rightarrow(1)$. Let $\gamma: C \rightarrow B$ be compatible with $\alpha$. Then $\gamma_{b}: X_{B} \rightarrow Y_{C}$ and $\alpha_{b}: X_{B} \rightarrow Y_{A}$ are compactifications of the same topological space. By (4), $Y_{A}$ is homeomorphic to $\beta X_{B}$, so there is a continuous map $\varphi: Y_{A} \rightarrow Y_{C}$ with $\varphi \circ \alpha_{b}=\gamma_{b}$.

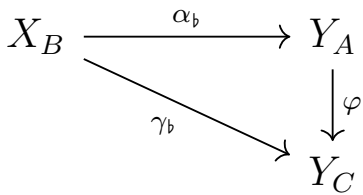

This implies that $\left(\alpha_{b}\right)^{b} \circ \varphi^{*}=\left(\gamma_{b}\right)^{b}$ since if $f \in C\left(Y_{C}\right)$, then

$$
\left[\left(\alpha_{b}\right)^{b} \circ \varphi^{*}\right](f)=f \circ \varphi \circ \alpha_{b}=f \circ \gamma_{b}=\left(\gamma_{b}\right)^{b}(f) .
$$

Define $\delta=\zeta_{A}^{-1} \circ \varphi^{*} \circ \zeta_{C}$. We have the following diagram.

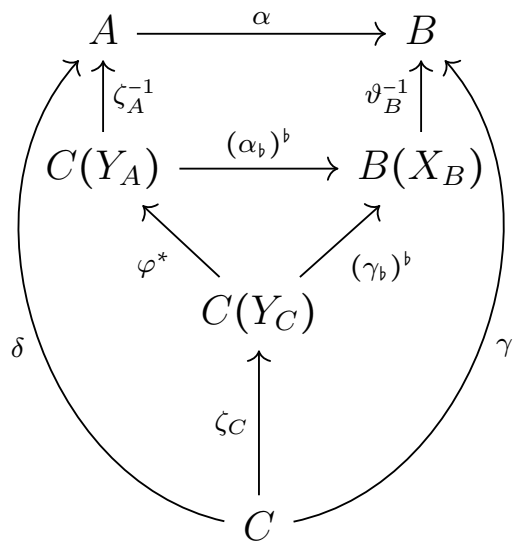

We just observed that the middle triangle commutes, and the top square commutes by Lemma 6.1. Another application of Lemma 6.1 yields that $\gamma=\vartheta_{B}^{-1} \circ\left(\gamma_{b}\right)^{b} \circ \gamma_{C}$. Thus, $\alpha \circ \delta=\gamma$, which proves that $\alpha$ is maximal.

$(4) \Rightarrow(5)$. By (4) we may assume $\alpha$ is the basic extension $\iota: C^{*}\left(X_{B}\right) \rightarrow B\left(X_{B}\right)$. Then $\alpha[A]=C^{*}\left(X_{B}\right)$. If $b \in B\left(X_{B}\right)$ is a meet from $C^{*}\left(X_{B}\right)$, then it is upper semicontinuous by [9, Lem. 4.1], and if it is a join from $C^{*}\left(X_{B}\right)$, then it is lower semicontinuous by the dual of [9, Lem. 4.1]. Therefore, if $b$ is both a join and meet from $C^{*}\left(X_{B}\right)$, then $b$ is continuous, so $b \in C^{*}\left(X_{B}\right)=\alpha[A]$. 
$(5) \Rightarrow(2)$. Let $f \in C^{*}\left(X_{B}\right)$. By Lemma 7.3, $f$ is both a pointwise join and a meet from elements of $\left(\alpha_{b}\right)^{*}\left[\zeta_{A}[A]\right]$. By $(5), \vartheta_{B}^{-1}(f) \in \alpha[A]$, so $f \in \vartheta_{B} \alpha[A]=\mu[A]$. Thus, $\mu$ is onto. Since it is $1-1$, we conclude that $\mu$ is an isomorphism.

As a consequence, we obtain that mbasic is a full subcategory of ubasic.

Definition 7.14. Let SComp be the full subcategory of Comp consisting of Stone-Cech compactifications.

Theorem 6.3 and Proposition 7.13 immediately yield the following:

Theorem 7.15. There is a dual equivalence between SComp and mbasic.

It is well known that CReg and SComp are equivalent (see, e.g., [6, Prop. 6.8]). Thus, as an immediate consequence we obtain:

Theorem 7.16. There is a dual equivalence between CReg and mbasic.

Remark 7.17. To describe the functors yielding the dual equivalence of Theorem 7.16, we recall that the equivalence between CReg and SComp is obtained by the functors S: CReg $\rightarrow$ SComp and F : SComp $\rightarrow$ CReg. The covariant functor $S$ sends a completely regular space $X$ to the Stone-Cech compactification $s: X \rightarrow \beta X$ and a continuous map $f: X \rightarrow Y$ to the unique continuous map $\beta f: \beta X \rightarrow \beta Y$ that makes the following diagram commute.

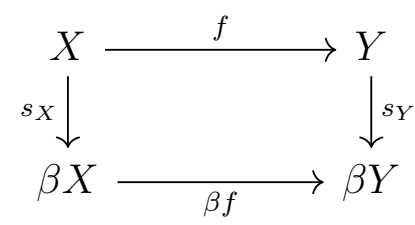

The covariant functor $\mathrm{F}$ sends a Stone-Čech compactification $s: X \rightarrow \beta X$ to $X$, and a morphism $(f, \beta f)$ to $f$. The dual equivalence of Theorem 7.16 is obtained by the contravariant functors $\mathrm{E} \circ \mathrm{S}$ and $\mathrm{F} \circ \mathrm{C}$. We give a more direct description of the contravariant functors between CReg and mbasic that yield this dual equivalence.

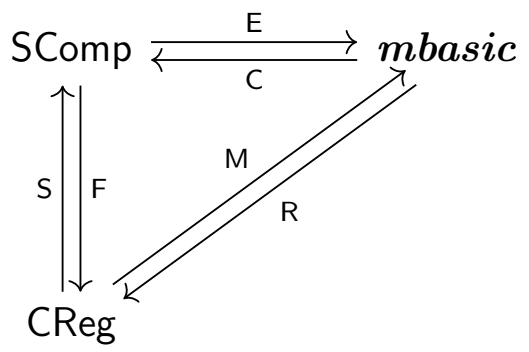

The contravariant functor $\mathrm{M}:$ CReg $\rightarrow$ mbasic sends a completely regular space $X$ to $\iota: C^{*}(X) \rightarrow B(X)$, and a continuous map $f: X \rightarrow Y$ to $\left(f^{*}, f^{+}\right)$.

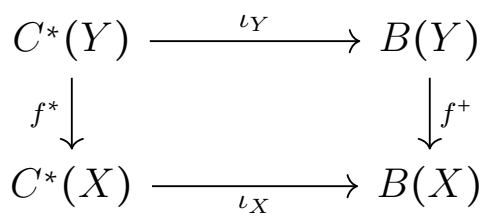


By Example 4.9(2) and Theorem 7.13, $\mathrm{M}$ is a well-defined functor. The contravariant functor $\mathrm{R}$ sends a maximal basic extension $\alpha: A \rightarrow B$ to $\left(X_{B}, \tau_{\alpha}\right)$, and a morphism $(\rho, \sigma)$ to $\sigma_{+}$: $X_{B^{\prime}} \rightarrow X_{B}$. By Theorem 5.5 and Lemma 5.8, $\mathrm{R}$ is a well-defined functor. That $\mathrm{F} \circ \mathrm{C}=\mathrm{S}$ follows from the definition of the functors, and $\mathrm{E} \circ \mathrm{S} \cong \mathrm{M}$ follows from Example 4.9)(2). Thus, the above diagram commutes.

We conclude the article by deriving several consequences of Theorem 7.16 , Recall that a completely regular space $X$ is strongly zero-dimensional if $\beta X$ is zero-dimensional (see, e.g., [10, Thms. 6.2.7 and 6.2.12]). We next obtain a duality for strongly zero-dimensional spaces.

Theorem 7.18. The dual equivalence between CReg and mbasic restricts to a dual equivalence between the full subcategory of CReg consisting of strongly zero-dimensional spaces and the full subcategory of mbasic consisting of the maximal basic extensions $\alpha: A \rightarrow B$ for which $A$ is a clean ring.

Proof. Let $X$ be a strongly zero-dimensional space. Then $\mathrm{M}(X)$ is the maximal extension $\iota: C^{*}(X) \rightarrow B(X)$. Since $X$ is strongly zero-dimensional, $C^{*}(X)$ is clean by [1, Thm. 2.5]. Let $\alpha: A \rightarrow B$ be a maximal extension with $A$ clean. Then the image under $\mathrm{R}$ of $\alpha$ is the completely regular space $X_{B}$. By Proposition 7.13, $Y_{A}$ is the Stone-Čech compactification of $X_{B}$. Since $A$ is clean, $Y_{A}$ is a zero-dimensional space [2, Thm. 5.9]. Thus, $X_{B}$ is strongly zero-dimensional. To complete the proof, apply Theorem 7.16 .

Theorem 7.19. The dual equivalence between CReg and mbasic restricts to a dual equivalence between the full subcategory of CReg consisting of extremally disconnected spaces and the full subcategory of mbasic consisting of the maximal extensions $\alpha: A \rightarrow B$ with $A \in \mathbf{d b a \ell}$.

Proof. If $X$ is an extremally disconnected space, then so is $\beta X$ (see, e.g., [10, Thm. 6.2.27]). The image under $\mathrm{M}$ of $X$ is the maximal extension $\iota: C^{*}(X) \rightarrow B(X)$. Since $C^{*}(X)$ is isomorphic to $C(\beta X)$, we see that $C^{*}(X) \in \boldsymbol{d} \boldsymbol{b a} \boldsymbol{\ell}$ by Corollary 2.6. Conversely, if $\alpha: A \rightarrow B$ is a maximal extension, then $A \cong C\left(Y_{A}\right)$, and if $A \in \boldsymbol{d} \boldsymbol{b a} \boldsymbol{\ell}$, then $Y_{A}$ is an extremally disconnected space by Corollary 2.6. By Proposition 7.13, $Y_{A}$ is the Stone-Cech compactification of $X_{B}$. Thus, $X_{B}$ is extremally disconnected (see, e.g., [10, Thm. 6.2.27]). Now apply Theorem[7.16,

Recall that a topological space $X$ is connected if $\varnothing, X$ are the only clopens of $X$, and that a commutative ring $A$ is indecomposable if $\operatorname{Id}(A)=\{0,1\}$.

Theorem 7.20. The dual equivalence between $\mathrm{CReg}$ and mbasic restricts to a dual equivalence between the full subcategory of CReg consisting of connected spaces and the full subcategory of mbasic consisting of the maximal extensions $\alpha: A \rightarrow B$ with $A$ an indecomposable ring.

Proof. Let $X$ be connected. The image under $\mathrm{M}$ of $X$ is the maximal extension $\iota: C^{*}(X) \rightarrow$ $B(X)$. The idempotents of $C^{*}(X)$ are exactly the characteristic functions of clopen subsets of $X$. Since $X$ is connected, the only clopen subsets are $\varnothing$ and $X$, so $\operatorname{Id}\left(C^{*}(X)\right)=\{0,1\}$, and hence $C^{*}(X)$ is indecomposable. Conversely, if $\alpha: A \rightarrow B$ is a maximal extension with $A$ indecomposable, then $A \cong C\left(Y_{A}\right)$ and $Y_{A}$ has no nontrivial clopen subsets. Therefore, $Y_{A}$ 
is connected. By Proposition 7.13, $Y_{A}$ is the Stone-Cech compactification of $X_{B}$. Thus, $X_{B}$ is connected (see, e.g., [10, Thm. 6.1.14]). Now apply Theorem 7.16.

\section{ACKNOWLEDGEMENTS}

We would like to thank the referee for careful reading and suggesting a more general statement of Lemma 7.2.

\section{REFERENCES}

[1] F. Azarpanah, When is $C(X)$ a clean ring?, Acta Math. Hungar. 94 (2002), no. 1-2, 53-58.

[2] G. Bezhanishvili, P. J. Morandi, and B. Olberding, Bounded Archimedean $\ell$-algebras and GelfandNeumark-Stone duality, Theory Appl. Categ. 28 (2013), Paper No. 16, 435-475.

[3] _ Dedekind completions of bounded Archimedean $\ell$-algebras, J. Algebra Appl. 12 (2013), no. 1, $16 \mathrm{pp}$.

[4] _ A functional approach to Dedekind completions and the representation of vector lattices and

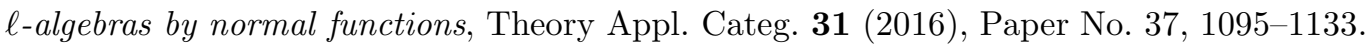

[5] _ Canonical extensions of bounded archimedean vector lattices, Algebra Universalis 79 (2018), no. 1, Paper No. 12, 17 pp.

[6] - An extension of de Vries duality to completely regular spaces and compactifications, Topology Appl. 257 (2019), 85-105.

[7] G. Birkhoff, Lattice theory, third ed., American Mathematical Society Colloquium Publications, vol. 25, American Mathematical Society, Providence, R.I., 1979.

[8] N. Dăneţ, Riesz spaces of normal semicontinuous functions, Mediterr. J. Math. 12 (2015), no. 4, 13451355.

[9] R. P. Dilworth, The normal completion of the lattice of continuous functions, Trans. Amer. Math. Soc. 68 (1950), 427-438.

[10] R. Engelking, General topology, second ed., Sigma Series in Pure Mathematics, vol. 6, Heldermann Verlag, Berlin, 1989, Translated from the Polish by the author.

[11] I. Gelfand and M. Neumark, On the imbedding of normed rings into the ring of operators in Hilbert space, Rec. Math. [Mat. Sbornik] N.S. 12(54) (1943), 197-213.

[12] L. Gillman and M. Jerison, Rings of continuous functions, The University Series in Higher Mathematics, D. Van Nostrand Co., Inc., Princeton, N.J.-Toronto-London-New York, 1960.

[13] M. Henriksen, J. R. Isbell, and D. G. Johnson, Residue class fields of lattice-ordered algebras, Fund. Math. 50 (1961/1962), 107-117.

[14] P. T. Johnstone, Stone spaces, Cambridge Studies in Advanced Mathematics, vol. 3, Cambridge University Press, Cambridge, 1982.

[15] W. A. J. Luxemburg and A. C. Zaanen, Riesz spaces. Vol. I, North-Holland Publishing Co., Amsterdam, 1971, North-Holland Mathematical Library.

[16] H. Nakano, Über das System aller stetigen Funktionen auf einem topologischen Raum, Proc. Imp. Acad. Tokyo 17 (1941), 308-310.

[17] M. H. Stone, Applications of the theory of Boolean rings to general topology, Trans. Amer. Math. Soc. 41 (1937), no. 3, 375-481.

[18] — A general theory of spectra. I, Proc. Nat. Acad. Sci. U. S. A. 26 (1940), 280-283.

[19] — Boundedness properties in function-lattices, Canadian J. Math. 1 (1949), 176-186. 
New Mexico State University

E-mail address: guram@nmsu.edu

New Mexico State University

E-mail address: pmorandi@nmsu.edu

New Mexico State University

E-mail address: bruce@nmsu.edu 